\title{
Isolated elliptical galaxies in the local Universe
}

\author{
I. Lacerna ${ }^{1,2,3}$, H. M. Hernández-Toledo ${ }^{4}$, V. Avila-Reese ${ }^{4}$, J. Abonza-Sane ${ }^{4}$, and A. del Olmo ${ }^{5}$
}

\author{
${ }^{1}$ Instituto de Astrofísica, Pontificia Universidad Católica de Chile, Av. V. Mackenna 4860, Santiago, Chile \\ e-mail: ialacern@astro.puc.cl \\ 2 Centro de Astro-Ingeniería, Pontificia Universidad Católica de Chile, Av. V. Mackenna 4860, Santiago, Chile \\ 3 Max Planck Institute for Astronomy, Königstuhl 17, 69117 Heidelberg, Germany \\ 4 Instituto de Astronomía, Universidad Nacional Autónoma de México, A.P. 70-264, 04510 México D. F., Mexico \\ 5 Instituto de Astrofísica de Andalucía IAA - CSIC, Glorieta de la Astronomía s/n, 18008 Granada, Spain
}

Received 26 November 2015 / Accepted 6 January 2016

\begin{abstract}
Context. We have studied a sample of 89 very isolated, elliptical galaxies at $z<0.08$ and compared their properties with elliptical galaxies located in a high-density environment such as the Coma supercluster.

Aims. Our aim is to probe the role of environment on the morphological transformation and quenching of elliptical galaxies as a function of mass. In addition, we elucidate the nature of a particular set of blue and star-forming isolated ellipticals identified here.

Methods. We studied physical properties of ellipticals, such as color, specific star formation rate, galaxy size, and stellar age, as a function of stellar mass and environment based on SDSS data. We analyzed the blue and star-forming isolated ellipticals in more detail, through photometric characterization using GALFIT, and infer their star formation history using STARLIGHT.

Results. Among the isolated ellipticals $\approx 20 \%$ are blue, $\$ 8 \%$ are star forming, and $\approx 10 \%$ are recently quenched, while among the Coma ellipticals $\approx 8 \%$ are blue and just $\lesssim 1 \%$ are star forming or recently quenched. There are four isolated galaxies $(\approx 4.5 \%)$ that are blue and star forming at the same time. These galaxies, with masses between $7 \times 10^{9}$ and $2 \times 10^{10} h^{-2} M_{\odot}$, are also the youngest galaxies with light-weighted stellar ages $\lesssim 1 \mathrm{Gyr}$ and exhibit bluer colors toward the galaxy center. Around $30-60 \%$ of their presentday luminosity, but only $<5 \%$ of their present-day mass, is due to star formation in the last $1 \mathrm{Gyr}$.

Conclusions. The processes of morphological transformation and quenching seem to be in general independent of environment since most of elliptical galaxies are "red and dead", although the transition to the red sequence should be faster for isolated ellipticals. In some cases, the isolated environment seems to propitiate the rejuvenation of ellipticals by recent $(<1 \mathrm{Gyr})$ cold gas accretion.
\end{abstract}

Key words. galaxies: elliptical and lenticular, $\mathrm{cD}$ - galaxies: formation - galaxies: fundamental parameters - galaxies: photometry galaxies: structure - galaxies: star formation

\section{Introduction}

An essential aspect in the theory of formation and evolution of galaxies is the understanding of the mechanisms behind their morphological transformation and quenching of star formation. Among the wide diversity of morphological types, elliptical (E) galaxies (so defined by Hubble 1926) seem to be those in the final stage of transformation to quiescent objects with regular and smooth structures (cf. Vulcani et al. 2015). As a result of their spheroidal and compact structure, supported against gravity by velocity dispersion, E galaxies have been proposed to be the result of major and minor mergers of disk galaxies, after which the gas would have been depleted and star formation quenched. The mergers could have happened early, when the disks were gaseous, or late, between gas-poor stellar galaxies (dry mergers). Moreover, in general nearby E galaxies are located in highdensity environments (Oemler 1974; Dressler 1980; Bamford et al. 2009), they have red colors and low values of specific star formation rates (i.e., they are quiescent), and their stellar population are mostly old with high metallicities and $\alpha / \mathrm{Fe}$ ratios (e.g., Roberts \& Haynes 1994; Kauffmann 1996; Kuntschner 2000; Baldry et al. 2004; Bell et al. 2004; Thomas et al. 2005; Kuntschner et al. 2010, for a review and more references see Blanton \& Moustakas 2009). All these facts motivated the idea that the 'red and dead' galaxies formed long ago by violent processes in a high-density environment that contributed to avoid further gas accretion into the galaxy.
Recent detailed observational studies have shown that ellipticals, in spite of the fact that they are the most regular galaxies, present more complex structures and more variations in their properties than previously thought (see, e.g., Blanton \& Moustakas 2009). A relevant question is how the properties of E galaxies vary with environment. Since the prevailing mechanism of $\mathrm{E}$ galaxy formation is that of major mergers (e.g., Hernquist 1993; Kauffmann 1996; Tutukov et al. 2007; Schawinski et al. 2014), which commonly happen in dense regions before they virialize, the environment is then expected to play a key role in the properties of ellipticals and their formation histories and quenching processes. However, it could be that local galaxy-halo processes and the mass scale rather than external processes are mostly responsible for the quenching and general properties of the post-merger systems. Hence, the study of isolated $\mathrm{E}$ galaxies is important since, in this case, the quenching mechanisms associated with the group/cluster environment (starvation, tidal, and ram-pressure gas stripping, etc.) are not acting. In general, isolated galaxies are optimal objects for constraining the internal physical processes that drive galaxy evolution.

The question of formation of E galaxies in isolated environments is on its own of great interest. Are these galaxies, for a given mass, different from those in clusters? Do both populations follow the same correlations with mass? Semianalytic models in the context of the popular $\Lambda$ cold dark matter $(\Lambda \mathrm{CDM})$ cosmology predict that on average the ellipticals formed in field haloes should have stellar ages comparable to those formed in rich 
clusters. However, in the field environment a more significant fraction of ellipticals with younger stellar populations is predicted than in clusters (Kauffmann 1996; Niemi et al. 2010). This may indicate different formation histories. Theoretical models suggest that ellipticals in clusters form through dissipative infall of gas and numerous mergers that took place at early epochs (5 to $10 \mathrm{Gyr}$ ago), whereas some field ellipticals form through recent major mergers and are still in the process of accreting cold gas.

In most of the previous observational works, early-type (E and S0/a) galaxies in general were studied. These works find that early-type galaxies are mostly red/passive, but there is also a fraction of blue/star-forming objects; this blue fraction increases as the mass is smaller and the environment is less dense (e.g., Schawinski et al. 2009; Kannappan et al. 2009; Thomas et al. 2010; McIntosh et al. 2014; Schawinski et al. 2014; Vulcani et al. 2015). The fraction of blue early-type galaxies at masses larger than $\log \left(M_{\mathrm{S}} / M_{\odot}\right) \approx 11$ is virtually null, showing that the most massive galaxies formed very early and efficiently quenched their growth by star formation. Interesting enough, the trends seen at $z \sim 0$ are similar at higher redshifts, although the fractions of blue early-type galaxies increase significantly with $z$ (Huertas-Company et al. 2010). For E galaxies in the field, which may include galaxies in loose and poor groups with dynamical masses $<10^{13} M_{\odot}$, it has been found that their colors are bluer on average and show more scatter in color than ellipticals in rich groups or clusters (de Carvalho \& Djorgovski 1992). Regarding pure E galaxies in very isolated environments, the samples in these studies are usually composed of only a few bright (massive) objects (e.g., Colbert et al. 2001; Marcum et al. 2004; Reda et al. 2004; Denicoló et al. 2005; Collobert et al. 2006; Hau \& Forbes 2006; Smith et al. 2010; Lane et al. 2013, 2015; Richtler et al. 2015; Salinas et al. 2015). In contrast to these works, Smith et al. (2004) and Stocke et al. (2004) presented relatively large samples of 32 and 65 isolated E galaxies, respectively, but they did not consider the radial velocity separation of companion galaxies to classify an isolated candidate.

In view of the shortage of observational samples of welldefined $\mathrm{E}$ (pure spheroidal) galaxies in extreme isolation, we present here a relatively complete sample of these galaxies in a large mass range and compare some of their properties to those ellipticals located in a high-density environment, the Coma supercluster. Our sample comes from the catalog of local isolated galaxies by Hernández-Toledo et al. (2010), which includes the redshift information creating a robust isolated sample of pure Es. We explore whether very isolated ellipticals differ in some photometric, spectroscopic, and structural properties with those of the Coma supercluster, and whether both isolated and highdensity ellipticals follow similar correlations with mass. Our final aim is to probe the role of environment on the morphological transformation and quenching of $E$ galaxies as a function of mass. Isolated ellipticals, whose results are very different from those in the cluster environment, are studied in more detail. In particular, we focus on a set of blue and star-forming (hereafter $\mathrm{SF}$ ) galaxies. In case the reason for their blue colors and recent star formation activity is due to a rejuvenation process produced by the recent accretion of cold gas, these isolated elliptical galaxies can be used as unique "sensors" of the gas cooling from the cosmic web.

The outline of the paper is as follows. The selection criteria of isolated galaxies along with the data set of galaxies in the Coma supercluster are described in Sect. 2. We present the results with the properties and mass dependences of $\mathrm{E}$ galaxies in Sect. 3. The implications of our results are discussed in
Sect. 4. The photometric and spectroscopic analysis of the particular subsample of blue and SF isolated elliptical galaxies is presented in Sect. 5. Finally, our conclusions are given in Sect. 6.

Throughout this paper we use the reduced Hubble constant $h$, where $H_{0}=100 \mathrm{~h} \mathrm{~km} \mathrm{~s}^{-1} \mathrm{Mpc}^{-1}$, with the following dependencies: stellar mass in $h^{-2} M_{\odot}$, absolute magnitude in $+5 \log (h)$, size and physical scale in $h^{-1} \mathrm{kpc}$, and halo mass in $h^{-1} M_{\odot}$, unless the explicit value of $h$ is specified.

\section{Data and selection criteria}

Our main goal is to study the properties of local elliptical galaxies in very isolated environments. For this, we use a particular galaxy sample described in Sect. 2.1. In order to compare some of the properties of these galaxies with those in a much denser environment, where ellipticals are more frequent, we use a compilation of elliptical galaxies in the Coma supercluster as described in Sect. 2.2.

\subsection{Isolated elliptical galaxies}

The isolated elliptical galaxies studied here come from the UNAM-KIAS catalog of Hernández-Toledo et al. (2010); this paper gives more details. Here we briefly refer to the sample and selection criteria.

The Sloan Digital Sky Survey (SDSS; York et al. 2000; Stoughton et al. 2002) produced two galaxy samples. One is a flux-limited sample to extinction corrected apparent Petrosian $r$-band magnitudes of 17.77 (the main galaxy sample), and a color-selected and flux-limited sample extending to $r_{\text {Pet }}=19.5$ (the luminous red galaxy sample). Galaxies with $r$-band magnitudes in the range $14.5 \leq r_{\text {Pet }}<17.6$ were selected from the DR4plus sample that is close to the SDSS Data Release 5 (Adelman-McCarthy et al. 2007). The survey region covers $4464 \mathrm{deg}^{2}$, containing 312338 galaxies. Hernández-Toledo et al. (2010) attempted to include brighter galaxies, but the spectroscopic sample of the SDSS galaxies is not complete for $r_{\text {Pet }}<14.5$. Thus, they searched in the literature and borrowed redshifts of the bright galaxies without SDSS spectra to increase the spectroscopic completeness. The final data set consists of 317533 galaxies with known redshift and SDSS photometry.

The isolation criteria is specified by three parameters. The first is the extinction-corrected Petrosian $r$-band apparent magnitude difference between a candidate galaxy and any neighboring galaxy, $\Delta m_{r}$. The second is the projected separation to the neighbor across the line of sight, $\Delta d$. The third is the radial velocity difference, $\Delta V$. Suppose a galaxy $i$ has a magnitude $m_{r, i}$ and $i$ band Petrosian radius $R_{i}$. It is regarded as isolated with respect to potential perturbers if the separation $\Delta d$ between this galaxy and a neighboring galaxy $j$ with magnitude $m_{r, j}$ and radius $R_{j}$ satisfies the conditions

$\Delta d \geq 100 \times R_{j}$

or $\Delta V \geq 1000 \mathrm{~km} \mathrm{~s}^{-1}$,

or the conditions

$\Delta d<100 \times R_{j}$

$\Delta V<1000 \mathrm{~km} \mathrm{~s}^{-1}$

$m_{r, j} \geq m_{r, i}+\Delta m_{r}$, 
for all neighboring galaxies. Here $R_{j}$ is the seeing-corrected Petrosian radius of galaxy $j$, measured in $i$-band using elliptical annuli to consider flattening or inclination of galaxies (Choi et al. 2007). Hernández-Toledo et al. (2010) chose $\Delta m_{r}=2.5$. Using these criteria, they found a total of 1548 isolated galaxy candidates. We note that a magnitude difference of 2.5 in this selection criteria translates into a factor of about 10 in brightness similar to that imposed by Karachentseva (1973).

In Hernández-Toledo et al. (2010), isolated elliptical galaxies were classified after some basic image processing and presented in mosaics of images including surface brightness profiles and the corresponding geometric profiles (ellipticity $\epsilon$, Position Angle PA and $A_{4} / B_{4}$ coefficients of the Fourier series expansions of deviations of a pure ellipse) from the $r$-band images to provide further evidence of boxy/disky character and other structural details. A galaxy was judged to be an elliptical if the $A_{4}$ parameter showed: 1) no significant boxy $\left(A_{4}<0\right)$ or disky $\left(A_{4}>0\right)$ trend in the outer parts; or 2$)$ a generally boxy $\left(A_{4}<0\right)$ character in the outer parts. We inspected for the presence/absence of 3) a linear component in the surface brightness-radius diagram. Morphologies were assigned according to a numerical code following the HyperLeda ${ }^{1}$ database convention; in particular for early-type galaxies, the following $T$ morphological parameters (Buta et al. 1994) are applied: -5 for E, -3 for E-S0, -2 for S0s, and 0 for S0a types. In the UNAM-KIAS sample there are 250 isolated early-type galaxies that satisfy $T \leq 0$ (E/S0), where 92 galaxies are ellipticals $(T \leq-4)$, which is $\approx 6 \%$ of the sample.

\subsection{Elliptical galaxies in the Coma supercluster}

To perform a comparative study of the isolated elliptical galaxies in the UNAM-KIAS catalog, we have compiled a sample of elliptical galaxies in a dense environment such as the Coma supercluster. This region is composed of the Coma and Leo clusters (Abell 1656 and Abell 1367, respectively) along with other galaxies in the filaments that connect these two rich clusters. To that purpose, we retrieve available data through the GOLDMine Database (Gavazzi et al. 2003). From the list of galaxies in the Coma supercluster ( 1000 objects), the quoted CGCG (Catalogue of Galaxies and of Clusters of Galaxies) principal name was used to cross-correlate with the HyperLeda database. In the latter catalog, 915 galaxies have morphological classification, where 131 objects correspond to pure elliptical galaxies. There are 113 elliptical galaxies with spectroscopic redshifts from the SDSS database using CasJobs ${ }^{2}$. We select galaxies in the redshift range of $4000<c z<9500 \mathrm{~km} \mathrm{~s}^{-1}$ as true members of the Coma supercluster (Gavazzi et al. 2014). With this, we obtain a sample of 102 elliptical galaxies with a mean redshift of $0.023 \pm 0.003$ and $r_{\text {Pet }}<15.4$. Figure 1 shows the spatial distribution of these galaxies in the Coma supercluster. Although some Es are not members of the two clusters, they probably belong to groups or regions in the outskirts of the clusters, which correspond to higher density environments compared to the low-density environment of isolated galaxies. Indeed, we checked that none of the elliptical galaxies in the Coma supercluster is classified as an isolated object by following Eqs. (1)-(5). In addition, we checked that the overall results and conclusions in this paper do not change if the velocity range is reduced (e.g., $6000<c z<8000 \mathrm{~km} \mathrm{~s}^{-1}$ ).

\footnotetext{
1 http://leda.univ-lyon $1 . \mathrm{fr} /$

2 See http://casjobs.sdss.org/CasJobs/
}

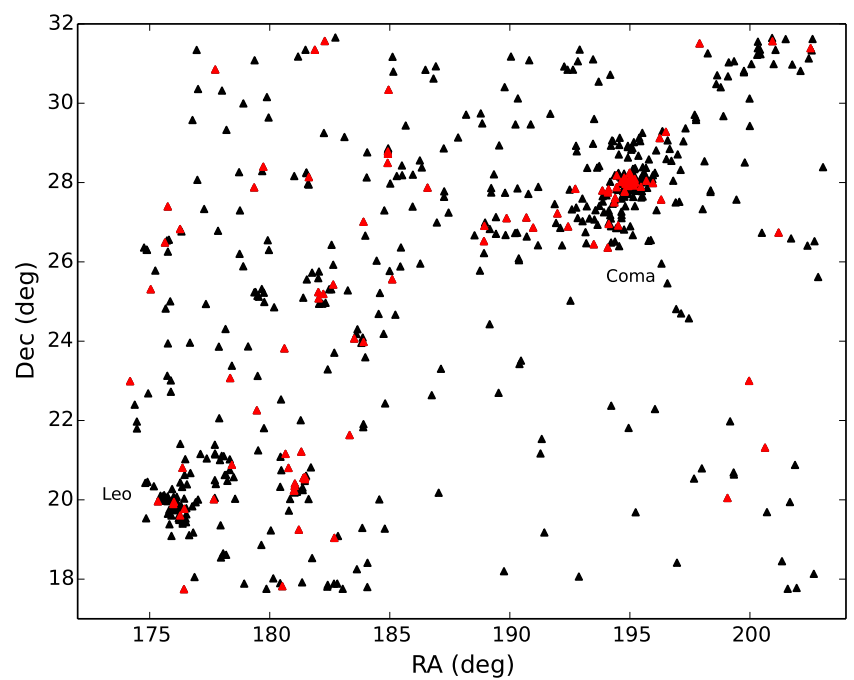

Fig. 1. Distribution in right ascension and declination of galaxies in the Coma supercluster with spectroscopic redshifts in the range $4000<$ $c z<9500 \mathrm{~km} \mathrm{~s}^{-1}$. Red points correspond to elliptical galaxies. Coma and Leo clusters (Abell 1656 and Abell 1367, respectively) are indicated in the plot.

\subsection{Physical properties}

Color measurements for the isolated galaxies and galaxies in the Coma supercluster were taken from the SDSS database with extinction corrected modelMag magnitudes (dered parameter in CasJobs). This magnitude is defined as the better of two magnitude fits: a pure de Vaucouleurs profile and a pure exponential profile.

We transform the SDSS colors to $B-R$ colors and the absolute magnitude in the $r$-band to the $R$-band in Sect. 3 following the equations, suggested by Niemi et al. (2010), written as

$B-R=(g-i)+0.44$

$M_{R}=M_{r}-0.35$.

We use the following stellar mass-to-light ratio of Bell et al. (2003) to estimate the stellar mass, $M_{\mathrm{s}}$, for the galaxy samples:

$$
\begin{aligned}
\log \left(M_{\mathrm{s}} / h^{-2} M_{\odot}\right)= & -0.306+1.097\left[{ }^{0.0}\left(g_{\text {Pet }}-r_{\text {Pet }}\right)\right] \\
& -0.1-0.4\left({ }^{0.0} M_{r_{\text {Pet }}}-5 \log (h)-4.64\right),
\end{aligned}
$$

where ${ }^{0.0}\left(g_{\text {Pet }}-r_{\text {Pet }}\right)$ is the color with Petrosian magnitudes (petroMag parameter in CasJobs), which are measured within a circular aperture defined by the shape of the light profile. In addition to the correction for Galactic extinction (Schlegel et al. 1998), we perform the $K$-correction and evolution correction at $z=0$ with kcorrect v4_2 (Blanton \& Roweis 2007). The Petrosian $r$-band absolute magnitude is ${ }^{0.0} M_{r_{\text {Pet }}}-5 \log (h)$, which is also $K$-corrected and evolution corrected at $z=0$. We include an extra correction to this absolute magnitude of -0.1 mag for elliptical galaxies since Petrosian magnitudes underestimate the total flux for these galaxies (Bell et al. 2003; McIntosh et al. 2014). The term -0.1 in Eq. (8) implies a Kroupa (2001) initial mass function (IMF). The systematic error is $0.10-0.15$ dex. The stellar mass estimation used here does not have systematic differences compared to other methods, for example, using spectral energy distribution fittings (see Dutton et al. 2011).

The specific star formation rate (sSFR) is simply defined as the star formation rate divided by the stellar mass. This quantity 
has been obtained from the MPA-JHU DR7 catalog 3 , which corresponds to an updated version of the estimates presented in Brinchmann et al. (2004) by using a spectrophotometric synthesis fitting model.

The radii used in this work, $R_{\mathrm{deV}}$, correspond to the de Vaucouleurs fit scale radius in the $r$-band (deVRad_r parameter in CasJobs). This radius is defined as the effective (halflight) radius of a de Vaucouleurs brightness profile, $I(r)=$ $I_{0} \mathrm{e}^{-7.67\left[\left(r / R_{\mathrm{deV}}\right)^{1 / 4}\right]}$.

Finally, the luminosity-weighted stellar age is obtained from the database of STARLIGHT ${ }^{4}$, through the population synthesis method developed by Cid Fernandes et al. (2005) and applied to the SDSS database.

\subsection{Halo masses}

We study how the properties of our isolated elliptical galaxies depend on the host halo mass. For this reason, we use the Yang et al. (2007, hereafter Y07) group catalog, which includes by construction the halo (virial) mass, $M_{\mathrm{h}}$, of galaxies down to some luminosity. This kind of halo-based group finders have emerged as a powerful method for estimating group halo masses, even when there is only one galaxy in the group. This method can recover, in a statistical sense, the true halo mass from mock catalogs with no significant systematics (Yang et al. 2008).

The halo mass in Y07 is based on either the characteristic stellar mass or the characteristic luminosity in the group. We use the halo mass based on the characteristic stellar mass, which is defined as the sum of the stellar mass of all the galaxies in the halo with ${ }^{0.1} M_{r}-5 \log (h) \leq-19.5$, where ${ }^{0.1} M_{r}$ is the $r$-band absolute magnitude with $K$-correction and evolution correction at $z=0.1$. They assume a one-to-one relation between the characteristic stellar mass and $M_{\mathrm{h}}$ by matching their rank orders for a given volume and a given halo mass function.

However, for single-galaxy groups, which are not complete in characteristic stellar mass, the halo mass estimates under the assumption of the one-to-one relation mentioned above are already not reliable. For this reason, the central galaxies that are fainter than the magnitude limit do not have halo mass estimates, where the halo mass lower limit in the Y07 catalog is $10^{11.6} h^{-1} M_{\odot}$. We point out that because of the method used in Y07, the estimated halo masses are not measurements of the true halo masses, but they are a very good statistical approximation.

\subsection{Redshift and stellar mass limits}

Since the UNAM-KIAS catalog reaches mainly out to $z=0.08$, throughout the text we use the redshift range $0<z<0.08$. Furthermore, in a magnitude limited sample, the minimum detected $M_{\mathrm{S}}$ depends on the redshift and on the stellar mass-toluminosity ratio, where the latter depends on galaxy colors. For the SDSS sample and its magnitude limit, van den Bosch et al. (2008, see also Yang et al. 2009) calculated the stellar mass limit at each $z$ above which the sample is complete. We adopt their limit as follows:

$$
\begin{aligned}
\log \left(M_{\mathrm{s}, \lim } / h^{-2} M_{\odot}\right)= \\
\quad \frac{4.852+2.246 \log \left(d_{L}\right)+1.123 \log (1+z)-1.186 z}{1-0.067 z} .
\end{aligned}
$$

\footnotetext{
3 Available at http://www . mpa-garching.mpg.de/SDSS/DR7/

4 http://www.starlight.ufsc.br
}

Our final sample of isolated ellipticals $(T \leq-4)$ consists of 89 galaxies using Eq. (9) (69 galaxies with halo mass estimates). For the average redshift of our sample of isolated E galaxies, $\bar{z}=0.037$, the corresponding average stellar mass limit is $\log \left(M_{\mathrm{s}, \lim } / h^{-2} M_{\odot}\right)=9.47$. We notice that only three E galaxies of these 89 have stellar masses smaller than this average mass limit.

On the other hand, we obtain a final sample of 102 elliptical galaxies in the Coma supercluster according to Eq. (9). The mass limit completeness at the average redshift of the Coma supercluster galaxies $(\bar{z}=0.023)$ is $\log \left(M_{\mathrm{s}, \lim } / h^{-2} M_{\odot}\right)=9.0$.

\section{Properties and mass dependences of elliptical galaxies}

\subsection{Colors and star formation rates}

Figure 2 shows the $(g-i)-M_{\mathrm{s}}$ diagram for our sample of isolated elliptical galaxies (black filled squares) and for ellipticals in the Coma supercluster (red filled triangles). The red solid line corresponds to the relation found by Lacerna et al. (2014) to separate red and blue galaxies, specifically

$(g-i)=0.16\left[\log \left(M_{\mathrm{s}}\right)-10\right]+1.05$,

where $M_{\mathrm{S}}$ is in units of $h^{-2} M_{\odot}$. As can be seen from the figure, most of the ellipticals located both in dense and isolated environments are red upon this criterion. However, there is a fraction of galaxies with bluer colors. We find that 18 isolated ellipticals ( $\approx 20 \%$ of the sample) are below the red line, some of them far away from this division line. Instead, there are only eight $(\approx 8 \%)$ ellipticals in Coma that are below the red line, and most of them are actually close to it, probably lying in what is called the green valley. Thus, the fraction of blue galaxies is higher in the isolated environment than in the Coma supercluster. For the blue isolated ellipticals, they become bluer as the mass is lower. At relatively low masses, $M_{\mathrm{s}}<10^{10.4} h^{-2} M_{\odot}$, the blue population only corresponds to the isolated elliptical sample. At high masses, most of the galaxies are red.

The top and right panels of Fig. 2 show the stellar mass and $g-i$ color distributions, respectively, for isolated elliptical galaxies (gray solid histogram) and elliptical galaxies located in the Coma supercluster (red open histogram). These normalized density distributions were obtained using the Knuth method for estimating the bin width implemented on astroML ${ }^{5}$ (Ivezić et al. 2014), which is also able to recognize substructure in data sets. The $g-i$ mean and median values of isolated and Coma supercluster ellipticals are similar, see Table 1, although the distribution is slightly narrower in the case of Coma galaxies (see also the difference among the 16th and 84th percentiles). Some isolated elliptical galaxies seem to be more massive than the most massive elliptical galaxies in the dense environment (e.g., NGC 4889 and NGC 4874 of Coma cluster), though the differences are within the mass uncertainties of 0.15 dex. We checked that our mass estimates for these galaxies are consistent with mass estimations based on spectral synthetic models from the MPA-JHU DR7 catalog.

Figure 3 shows sSFR as a function of stellar mass. We include as a red solid line the relation found by Lacerna et al. (2014) to separate passive and SF galaxies, i.e.,

$\log (\mathrm{s} S F R)=-0.65\left[\log \left(M_{\mathrm{s}}\right)-10\right]-10.87$,

http://www. astroml.org/ 
I. Lacerna et al.: Isolated elliptical galaxies in the local Universe

Table 1. Physical properties of isolated ellipticals and E galaxies in the Coma supercluster.

\begin{tabular}{|c|c|c|c|c|c|c|c|c|c|c|}
\hline \multirow[b]{2}{*}{ Property } & \multicolumn{5}{|c|}{ Isolated } & \multicolumn{5}{|c|}{ Coma } \\
\hline & Mean & $\sigma$ & Median & 16th & 84th & Mean & $\sigma$ & Median & 16th & 84th \\
\hline $\log \left(M_{\mathrm{S}}\right)^{a}$ & 10.55 & 0.46 & 10.62 & 10.17 & 10.95 & 10.55 & 0.29 & 10.51 & 10.24 & 10.84 \\
\hline$g-i$ & 1.18 & 0.10 & 1.19 & 1.09 & 1.28 & 1.20 & 0.05 & 1.20 & 1.14 & 1.24 \\
\hline $\log (\mathrm{sSFR})^{b}$ & -11.88 & 0.52 & -12.00 & -12.27 & -11.61 & -12.11 & 0.30 & -12.17 & -12.36 & -11.87 \\
\hline${ }^{0.0} M_{R}$ & -21.87 & 1.06 & -22.02 & -22.77 & -20.95 & -21.78 & 0.69 & -21.71 & -22.51 & -21.05 \\
\hline$B-R$ & 1.62 & 0.10 & 1.63 & 1.53 & 1.72 & 1.64 & 0.05 & 1.64 & 1.58 & 1.68 \\
\hline $\log \left(R_{\mathrm{deV}}\right)^{c}$ & 0.53 & 0.25 & 0.55 & 0.29 & 0.76 & 0.50 & 0.20 & 0.50 & 0.33 & 0.69 \\
\hline $\log (\text { age })^{d}$ & 9.63 & 0.30 & 9.67 & 9.42 & 9.86 & 9.72 & 0.20 & 9.74 & 9.59 & 9.88 \\
\hline
\end{tabular}

Notes. For each property, the columns correspond to the mean, its standard deviation, the median, the 16th, and 84 th percentiles. ${ }^{(a)} \log _{10}$ of the stellar mass in units of $h^{-2} M_{\odot}{ }^{(b)} \log _{10}$ of the specific star formation rate in units of $\mathrm{yr}^{-1} \cdot{ }^{(c)} \log _{10}$ of the radius of a de Vaucouleurs fit in the $r$-band in units of $\mathrm{kpc}(h=0.7) .{ }^{(d)} \log _{10}$ of the light-weighted stellar age in units of yr.

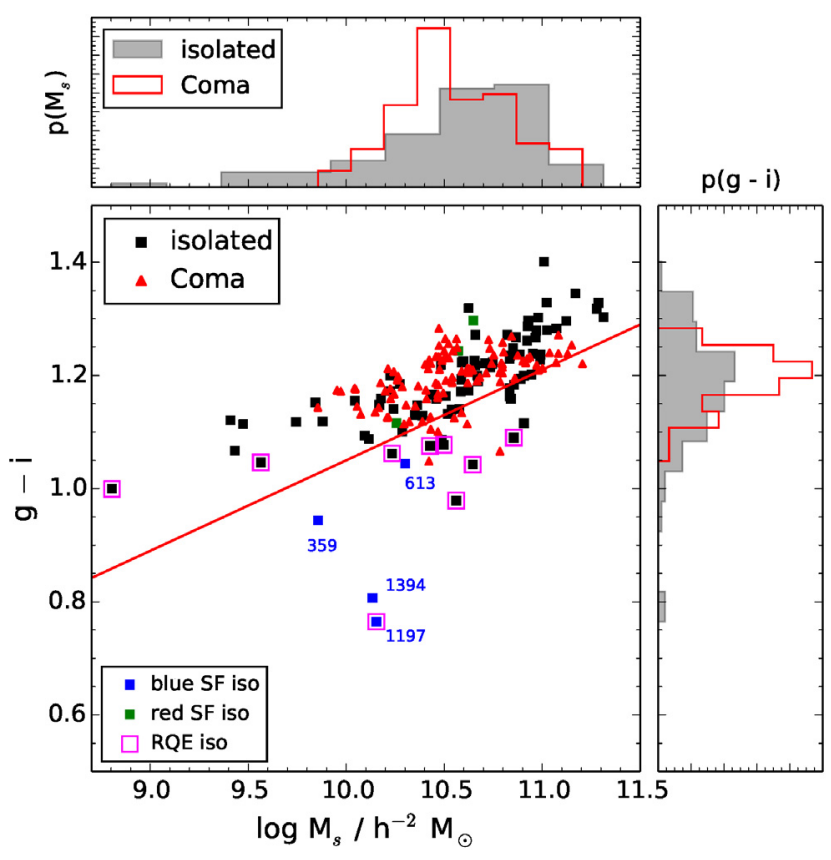

Fig. 2. As a function of stellar mass, $g-i$ color for our sample of isolated elliptical $(T \leq-4)$ galaxies out to $z=0.08$ (black filled squares). In addition, we include a sample of elliptical galaxies located in the Coma supercluster (red filled triangles). The red line shows Eq. (10) to separate red/blue galaxies. Blue filled squares and green filled squares show the blue and red isolated ellipticals, which are also star-forming galaxies according to Eq. (11), respectively. The numbers are the ID in the UNAM-KIAS catalog for the former. Magenta open squares correspond to the recently quenched elliptical (RQE) galaxies following the color-color criterion of Sect. 3.3. Top and right panels: normalized density distributions of stellar mass and $g-i$ color for isolated elliptical galaxies (gray solid histogram) and elliptical galaxies located in the Coma supercluster (red open histogram), respectively. The integral of each histogram sums to unity.

where $M_{\mathrm{S}}$ is in units of $h^{-2} M_{\odot}$ and sSFR is in units of $\mathrm{yr}^{-1}$. According to this relation, galaxies located above and below this line are considered SF and passive galaxies, respectively. Most of our isolated ellipticals are passive galaxies in terms of their star formation. In Figs. 2, 3, and the figures that follow, we plot with a blue (green) solid square those ellipticals that are blue (red) and SF galaxies; the magenta open squares highlight the "recently quenched ellipticals" to be described below. There are four blue SF ellipticals and three red SF galaxies. In total, we have seven SF isolated ellipticals $(\approx 8 \%$ of all the

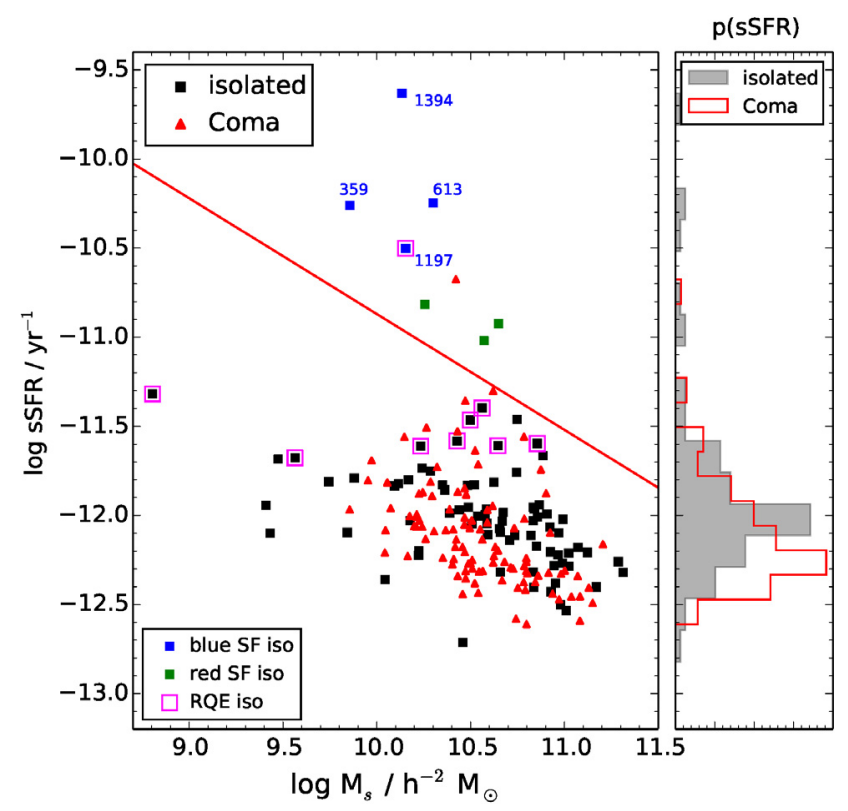

Fig. 3. Specific star formation rate (sSFR) as a function of the stellar mass. The symbols for the galaxies are the same as Fig. 2. The red line shows Eq. (11) to separate star-forming and passive galaxies (above and below the line, respectively). Right panel: normalized density distribution of sSFR for isolated elliptical galaxies (gray solid histogram) and elliptical galaxies located in the Coma supercluster (red open histogram). The integral of each histogram sums to unity.

isolated ellipticals). In Sect. 3.5, we see that at least one blue SF galaxy could be classified as AGN/LINER in the BPT diagram (Baldwin et al. 1981) and in Appendix A that other blue SF galaxy could be an AGN because of its broad component in $\mathrm{H} \alpha$. On the other hand, nearly all the E galaxies from the Coma supercluster (red filled triangles) are passive objects according to the above criterion. There is only one $(\$ 1 \%)$ elliptical in Coma that would be a SF galaxy.

In general, the distribution of isolated and Coma ellipticals in the $\mathrm{S} S F R-M_{\mathrm{s}}$ plane seems to be not too different, except for the small fraction of isolated ellipticals at masses $10^{9.7}<M_{\mathrm{S}}<$ $10^{10.7} h^{-2} M_{\odot}$ with relevant signs of star formation activity. The right panel of Fig. 3 shows the sSFR distributions for isolated elliptical galaxies (gray solid histogram) and elliptical galaxies in the Coma supercluster (red open histogram). The latter are slightly more passive, by $\sim 0.2$ dex according to the mean and median values reported in Table 1. 


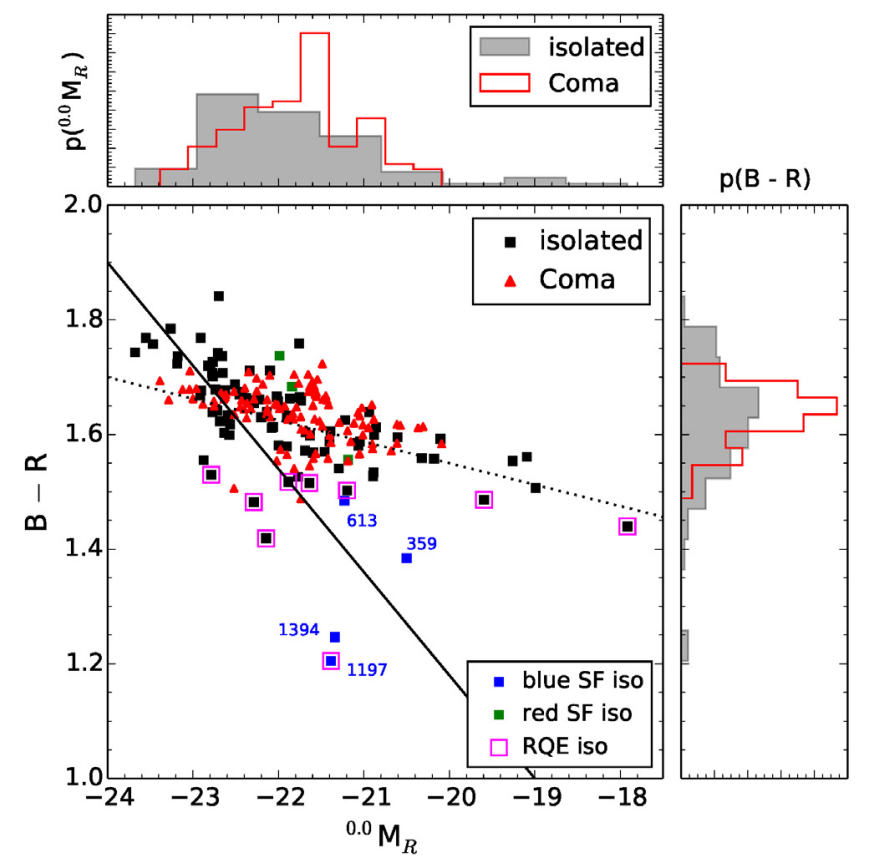

Fig. 4. $B-R$ color as a function of the absolute magnitude in the $R$-band ( $h=0.7, K$-corrected at $z=0$ ). The symbols for the galaxies are the same as Fig. 2. The linear fit of Niemi et al. (2010) for their (nonisolated) elliptical galaxies from a semianalytic model is shown as a dotted line. The fit for their simulated isolated elliptical galaxies is shown as a solid line. Top and right panels: normalized density distributions of absolute magnitude and $B-R$ color for isolated elliptical galaxies (gray solid histogram) and elliptical galaxies located in the Coma supercluster (red open histogram), respectively. The integral of each histogram sums to unity.

\subsection{The color-magnitude diagram}

It is well known that, in general, more luminous galaxies tend to have redder colors. In Fig. 4, as an example of the colormagnitude diagram (CMD), we plot the $B-R$ color against $R$-band absolute magnitude for our samples of isolated and Coma E galaxies. The symbols for the E galaxies are the same as in Fig. 2. The trend that galaxies are redder as their luminosity increases is followed by both isolated and Coma $\mathrm{E}$ galaxies. This means that ellipticals exhibit roughly a similar CMD regardless of the environment. However, as in the case of the color $-M_{\mathrm{s}}$ diagram, there is a small fraction of isolated ellipticals that follow a different trend, toward bluer colors as magnitudes are fainter. They show a steeper color-magnitude relation than the rest of the ellipticals, giving rise to a branch that systematically detaches from the red sequence. The blue SF galaxies identified in the previous figures (blue filled squares) are namely at the end of this branch.

The right panel of Fig. 4 shows the $B-R$ color distribution for isolated E galaxies (gray solid) and Es in the Coma supercluster (red open). The mean and median $B-R$ colors (reported in Table 1) are only slightly different between both samples. Elliptical galaxies in Coma are on average redder by $\sim 0.02 \mathrm{mag}$ than those in isolation, but the distribution of the former is narrower than that of the latter (see also the 16th and 84th percentiles). The top panel of this figure corresponds to the distribution of the absolute magnitude in the $R$-band. The mean and median values are reported in Table 1 . A slight bias is seen in the distribution toward brighter isolated ellipticals than in Coma.

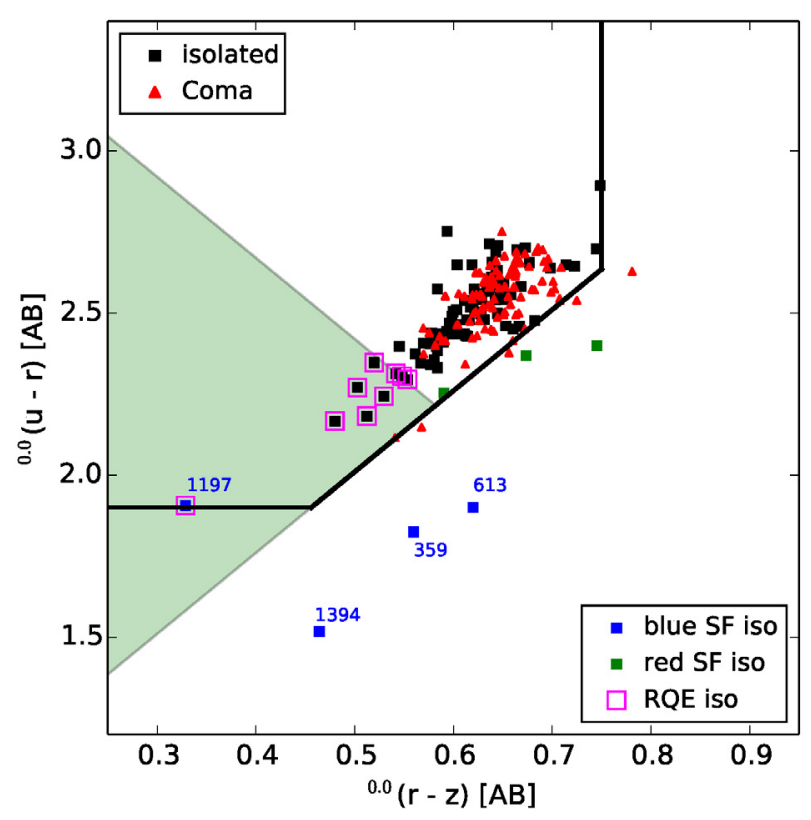

Fig. 5. Color-color diagram to identify recently quenched elliptical (RQE) galaxies. The colors are in $\mathrm{AB}$ magnitudes and $K$-corrected at $z=0$. The symbols for the galaxies are the same as Fig. 2. The thick black lines enclose the non-star-forming region of McIntosh et al. (2014) based on Holden et al. (2012). The RQE selection region is empirically defined as the green shaded triangle by McIntosh et al. (2014). Nine isolated elliptical galaxies are inside this region and are shown in magenta open squares.

\subsection{Recently quenched ellipticals and stellar ages}

McIntosh et al. (2014) introduced some criteria to define the population of ellipticals that have been quenched recently. These recently quenched ellipticals (hereafter RQE) are mostly blue, have light-weighted stellar ages shorter than $3 \mathrm{Gyr}$ (according to age determinations by Gallazzi et al. 2005), and lack of detectable emission for star formation. Such galaxies clearly experienced a recent quenching of star formation and are now transitioning to the red sequence. McIntosh et al. (2014) argue that these RQEs "have recent star formation histories that are distinct from similarly young and blue early-type galaxies with ongoing star formation (i.e., rejuvenated early-type galaxies)", and given their supra solar metallicities, they "are consistent with chemical enrichment from a significant merger-triggered star formation event prior to the quenching". The authors conclude that RQEs are strong candidates for 'first generation' ellipticals formed in a relatively recent major spiral-spiral merger. McIntosh et al. (2014) found an empirical criterion in the $(u-r)-(r-z)$ diagram to select RQEs.

Figure 5 shows the $(u-r)-(r-z)$ diagram for the isolated and Coma E galaxies. The green shaded triangle is the empirical region where RQEs lie according to McIntosh et al. (2014). There are nine $(\approx 10 \%)$ isolated E galaxies that are RQEs according to this criterion; they are shown with magenta open squares in Fig. 5 and in other figures. Among these nine ellipticals, seven are blue and two are red, according to Eq. (10), as can be seen in Fig. 2, and eight are passive, according to Eq. (11), as can be seen in Fig. 3. In Sect. 3.5 we see that the (marginal) RQE, which is a blue SF galaxy (highlighted with a blue solid square; UNAM-KIAS 1197), can instead be classified as a LINER according to the BPT diagram. There are no isolated RQEs more massive than $M_{\mathrm{S}} \approx 7 \times 10^{10} h^{-2} M_{\odot}$ (see, e.g., Fig. 2), and they reside in haloes of masses $₫ 6 \times 10^{12} h^{-1} M_{\odot}$ (see Fig. 9 below), 


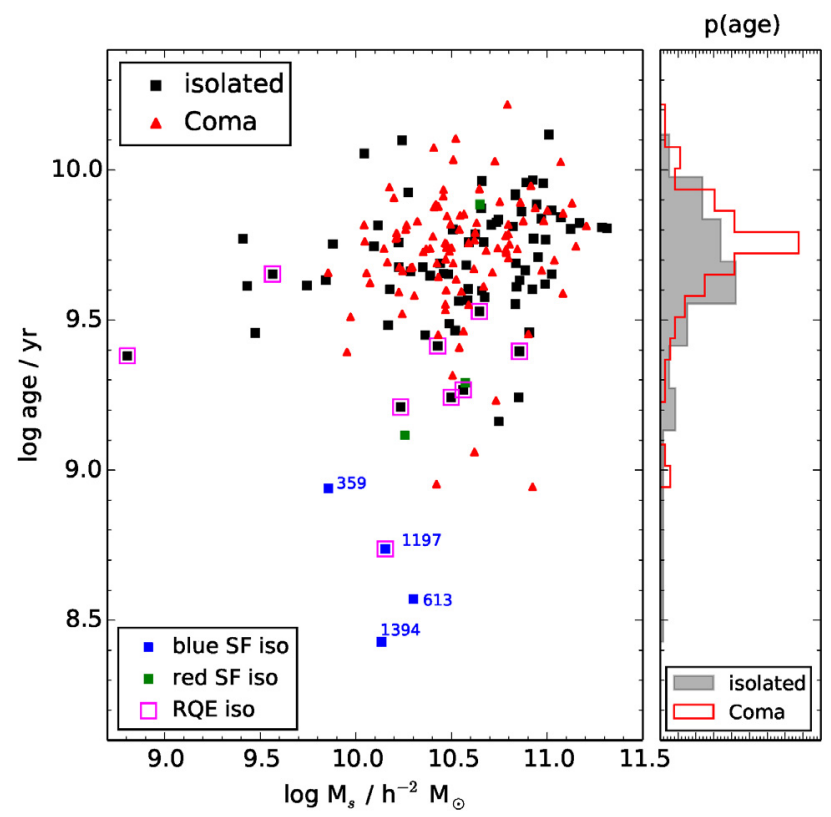

Fig. 6. Luminosity-weighted stellar age as a function of stellar mass. The symbols for the galaxies are the same as Fig. 2. Right panel: normalized density distribution of the luminosity-weighted stellar age for isolated elliptical galaxies (gray solid histogram) and elliptical galaxies in the Coma supercluster (red open histogram). The integral of each histogram sums to unity.

confirming that the most massive ellipticals were quenched long ago. The two RQEs classified as red galaxies are in fact the least massive of the nine RQEs; see Fig. 2.

In the case of the ellipticals in the Coma supercluster, there is only one RQE candidate although it lies in the lower border limit. Thus, while in the Coma supercluster there are virtually no RQEs (even those that are blue in Fig. 2 seem to have been quenched relatively early), RQEs are $\approx 10 \%$ among isolated $\mathrm{E}$ galaxies. This environmental difference is consistent with McIntosh et al. (2014), where the authors find that the vast majority of their RQEs are central in smaller groups $\left(M_{\mathrm{h}} \lesssim 3 \times 10^{12} h^{-1} M_{\odot}\right)$, i.e., they do not reside in high-density environments.

The black solid lines in Fig. 5 delimit the region of spectroscopically quiescent, red-sequence early-type galaxies according to Holden et al. (2012) and extended to $(u-r)=1.9$ in McIntosh et al. (2014). Galaxies outside this boundary are defined as pure star-forming (BPT H II emission). Most of the ellipticals in Coma (triangles) and isolated ellipticals (squares) qualify as non-SF systems in this diagram. There is a rough agreement between those galaxies defined as SF in Figs. 3 and 5. Blue SF isolated E galaxies (blue squares with numbers corresponding to their ID in the UNAM-KIAS catalog) are located far away from the non-SF region, whereas red SF isolated E galaxies (green squares) are close to the border.

In contrast to properties such as color and size (see Sect. 3.4), the luminosity-weighted stellar age does not show a significant dependence on stellar mass as shown in Fig. 6. From this figure it is clear that the blue SF isolated ellipticals are the youngest galaxies with luminosity-weighted stellar ages $\lesssim 1$ Gyr. This could indicate recent processes of star formation for this particular class of objects. The right panel shows the stellar age distribution of isolated ellipticals (gray solid histogram) and elliptical galaxies located in the Coma supercluster (red open histogram). The mean and median values are reported in Table 1. Ellipticals in Coma appear to be older than isolated ellipticals in general

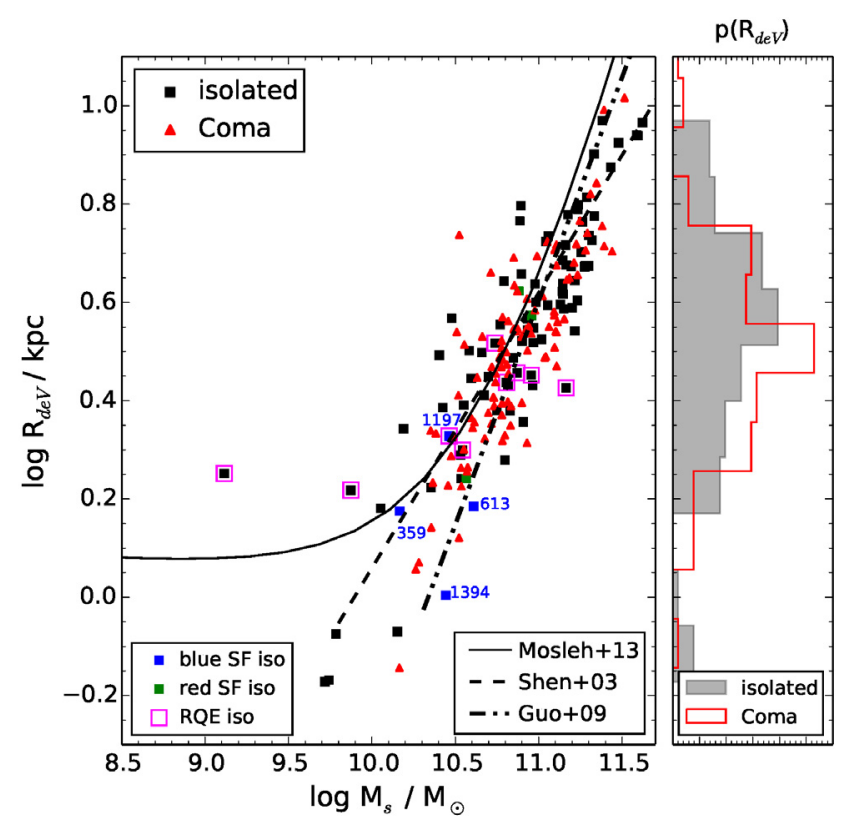

Fig. 7. Effective radius of a de Vaucouleurs fit, $R_{\mathrm{deV}}$, in the $r$-band as a function of stellar mass $(h=0.7)$. The symbols for the galaxies are the same as Fig. 2. We also include the mass-size relations of earlytype galaxies by Shen et al. (2003, dashed line), Guo et al. (2009, dotdot-dashed line) and Mosleh et al. (2013, solid line). Right panel: normalized density distribution of the radius for isolated elliptical galaxies (gray solid histogram) and elliptical galaxies in the Coma supercluster (red open histogram). The integral of each histogram sums to unity.

( $\sim 1$ Gyr older), although this difference is within the statistical uncertainties.

\subsection{Galaxy sizes}

Figure 7 shows the effective radius in the $r$-band for the different samples of elliptical galaxies as a function of stellar mass. The general trend that massive galaxies are bigger with no apparent dependence on environment is observed. Blue SF isolated ellipticals are smaller than $2.5 \mathrm{kpc}$. In the case of RQEs, they have $R_{\mathrm{deV}} \lesssim 3.5 \mathrm{kpc}$. The right panel of Fig. 7 shows the radius distribution of the isolated and Coma supercluster ellipticals (gray solid and red open histograms, respectively). We see that the radius distribution of Coma and isolated ellipticals are similar. If any, the former is slightly shifted to smaller radii with respect to the latter. The mean and median values of the radii are reported in Table 1. On average the radius for both samples are similar within the uncertainties, although the distribution of $\mathrm{E}$ galaxies in Coma is narrower than that of isolated ellipticals (e.g., see the 16 th and 84 th percentiles).

In Fig. 7 we also include the size-mass relation of earlytype galaxies $(n>2.5)$ reported in Shen et al. $(2003,2007)$ as a dashed line. They use the half-light radius in the $z$-band to define the size of their galaxies with $z \geq 0.005$. The dot-dot-dashed line shows the size-mass relation found by Guo et al. (2009) for early-type galaxies (usually $n>3.5$ ) at $z \leq 0.08$. They define the size as the half-light radius in the $r$-band. The solid line is the relation found by Mosleh et al. (2013) for their sample of earlytype galaxies between $0.01<z<0.02$. The morphology of these galaxies was obtained from the Galaxy Zoo Catalogue (Lintott et al. 2011); the size is also defined as the half-light radius in the $r$-band. Despite the fact that different size, morphological definitions, and samples were used in each one of these works, we find a qualitative good agreement among their studies and this 


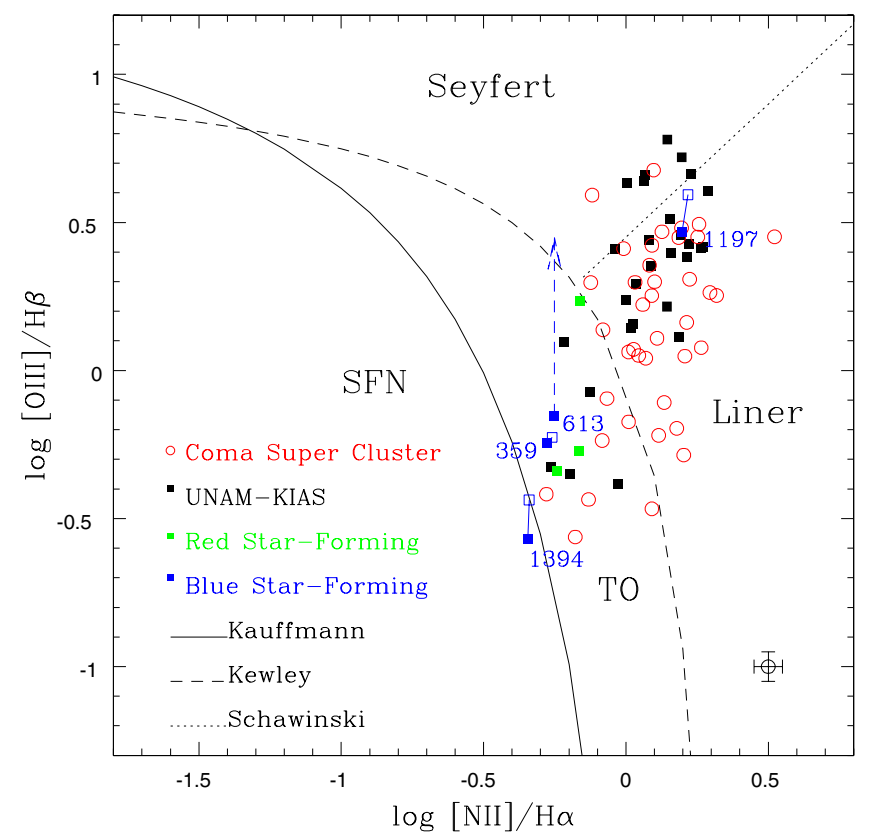

Fig. 8. BPT diagram for the isolated elliptical galaxies (black filled squares) of the UNAM-KIAS catalog and elliptical galaxies in the Coma supercluster (red open circles) with estimates from the STARLIGHT database. The typical uncertainty is indicated with the black open symbol with error bars. Blue and green solid points represent the blue SF and red SF isolated E galaxies, respectively. Open blue squares indicate particular measurements for blue SF isolated galaxies using the method described in Appendix C. Solid blue lines just connect the two different measurements for each blue SF elliptical, where the numbers correspond to their ID in the UNAM-KIAS catalog. Galaxies are classified as star-forming nuclei (SFN), transition objects (TO), or AGN (Seyfert/LINER) according to the relations of Kauffmann et al. (2003, solid line), Kewley et al. (2001, dashed line), and Schawinski et al. (2007, dotted line). The dashed arrow indicates that UNAM-KIAS 613 is rather a Seyfert 1.8 because of its broad components in $\mathrm{H} \alpha$ and $\mathrm{H} \beta$ (see details in Appendix A).

work at masses $M_{\mathrm{s}}>3 \times 10^{10} M_{\odot}$. The best agreement is obtained with the result by Guo et al. (2009). Their sample is more similar to ours (redshift limit, size definition, and more stringent criteria to select early-type systems). At lower masses, Mosleh et al. (2013) find a flatter size-mass relation. This is roughly followed by some of our low-mass isolated galaxies, although other low-mass ellipticals follow an extension of the trend exhibited by the massive galaxies. It seems that the size-mass relation is different or not unique at the low-mass end.

\subsection{BPT diagram}

A nuclear classification was carried out from diagrams in the optical diagnosis initially introduced by Baldwin et al. (1981, BPT) and redefined by Veilleux \& Osterbrock (1987). The BPT diagrams have been massively used for discriminating between different mechanisms of ionization and production of emission lines, separating the production mechanisms either by photoionization by massive OB stars (typical of regions with star formation) or nonstellar sources such as the presence of AGN in the core. We have cross-matched our sample of isolated and Coma supercluster E galaxies with the database of STARLIGHT, where the stellar population synthesis analysis developed by Cid Fernandes et al. (2005) was applied to SDSS galaxies.

Figure 8 shows the BPT diagram for the isolated E galaxies with reported lines in the STARLIGHT database (solid squares).
Those with reported lines correspond to 35 out of $89(\approx 39 \%)$ isolated $\mathrm{E}$ galaxies. The rest are probably quenched and/or without an AGN at present day. Out of the 35 galaxies, 24 are AGNs (19 LINERS and five Seyferts) and ten are transition objects (TOs) ${ }^{6}$. Only one galaxy (UNAM-KIAS 1394) is a SF nuclear (SFN) object. This galaxy is also a blue SF object as defined by us. Other two blue SF isolated Es are TOs, and one blue SF galaxy seems to be actually a LINER (UNAM-KIAS 1197). In Appendices $\mathrm{A}$ and $\mathrm{C}$, we describe a more detailed spectroscopical analysis of these four blue SF isolated ellipticals, using lines with $S / N>7$. Our results (blue open squares) are similar to those from the STARLIGHT database (blue solid squares). In the case of UNAM-KIAS 613, we find that this galaxy is actually a Seyfert 1.8 because of its broad components in $\mathrm{H} \alpha$ and $\mathrm{H} \beta$ (see details in Appendix A). Therefore, 25 isolated Es correspond to AGNs, nine are TOs and one galaxy is a SFN.

In the case of the E galaxies located in the Coma supercluster (red open circles), 39 out of $102(\approx 38 \%)$ have reported lines in the STARLIGHT database. The rest are probably quenched and without an AGN by today. Twenty-nine galaxies are LINERs, two are Seyferts and eight are TOs.

In general, there is a similar fraction of isolated and Coma supercluster ellipticals that present ionization emission lines associated with star formation or AGN activity. Among these, the fraction of those classified as SFN/TO is slightly larger for the isolated ellipticals than for the Coma ellipticals (11\% vs. $8 \%$ ). Thus, environment seems to have a weak influence on the fractions of ellipticals with a mixture of emission coming from a circumnuclear SF region and central low-luminosity AGN in the BPT diagram.

\section{Interpretation and implications}

The results presented above are consistent with several previous works on early-type galaxies mentioned in the Introduction. Our sample is different from previous samples in that it selects very isolated environments and refers only to morphologically well-defined elliptical galaxies $(T \leq-4)$ in a wide mass range. This allows us to probe the morphological transformation and quenching of the star formation as a function of mass, hopefully free of environmental effects.

The work by Vulcani et al. (2015) is the closest to the present paper. Using an automatic tool designed to attempt to reproduce the visual classifications (MORPHOT; Fasano et al. 2012), these authors made an effort to distinguish pure $\mathrm{E}$ galaxies from $\mathrm{S} 0 / \mathrm{Sa}$ galaxies in a sample complete above $\log \left(M_{\mathrm{S}} / M_{\odot}\right)=10.25$ at their redshift upper limit $z=0.11$. For those galaxies that Vulcani et al. (2015) call singles (no neighbors with a projected mutual distance of $0.5 h^{-1} \mathrm{Mpc}$ and a redshift within $1500 \mathrm{~km} \mathrm{~s}^{-1}$ ), $24 \pm 3 \%$ are ellipticals. Out of them, $\approx 83 \%$ are red according to their definition. The rest are blue and green (roughly $13 \%$ and $4 \%$, respectively). In our sample, the fraction of blue isolated ellipticals (we separate them only into blue and red galaxies) above $\log \left(M_{\mathrm{s}} / h^{-2} M_{\odot}\right)=9.94$ ( 10.25 for $h=0.7)$ is $\approx 21 \%$, which is slightly larger than in Vulcani et al. (2015), but yet within their error bars.

According to our analysis, the colors, sSFR, sizes, and luminosity-weighted ages of very isolated $\mathrm{E}$ galaxies are only slightly different from those in the Coma supercluster

6 The interpretation of TO objects as SF-AGN composites is not clear.
For example, McIntosh et al. (2014) discuss that these galaxies may be
neither SF nor an AGN, rather their emission may be dominated by the same non-nuclear ionization sources as many LINERs. 

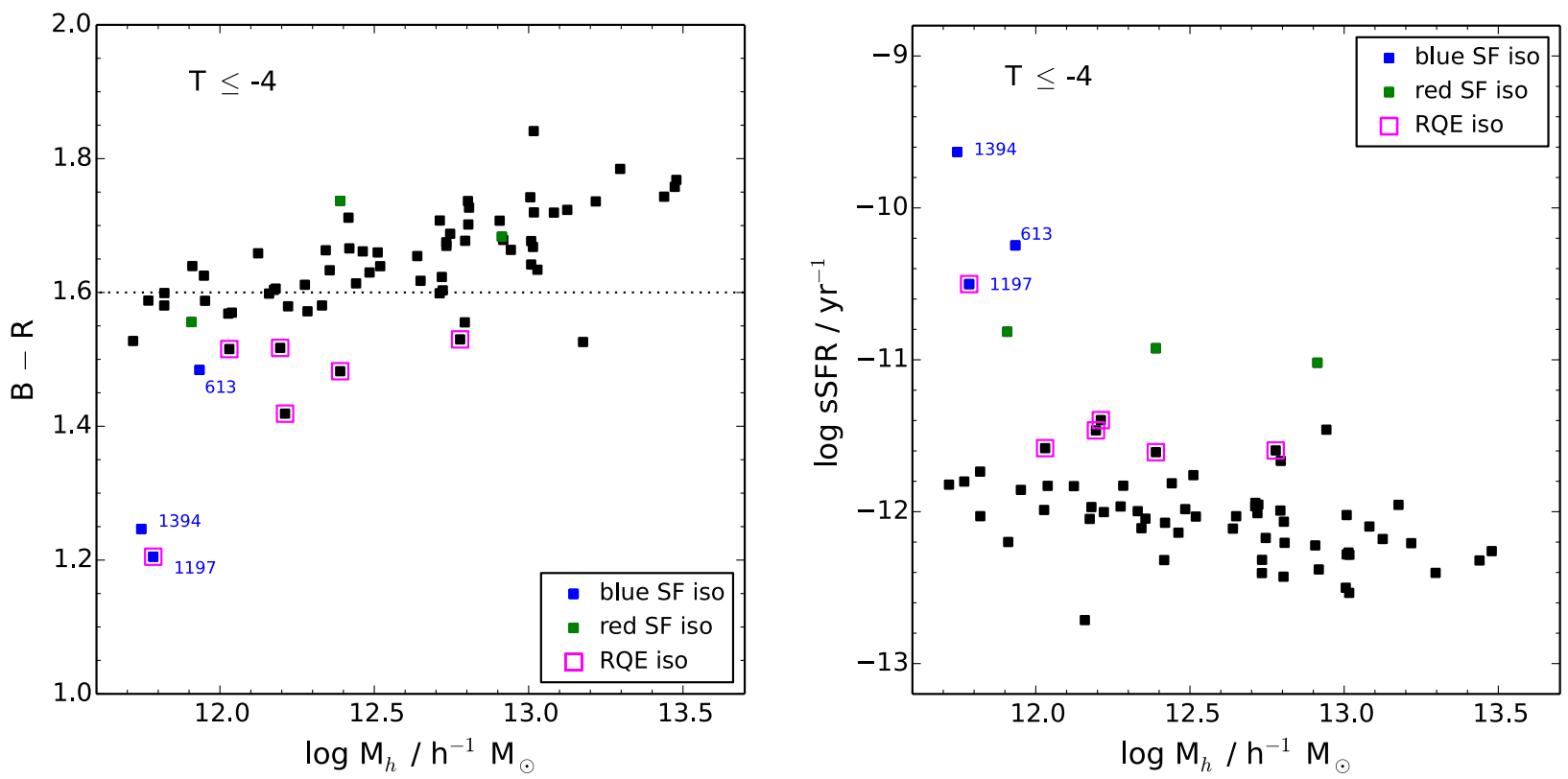

Fig. 9. $B-R$ color (left panel) and sSFR (right panel) as a function of the halo mass of isolated elliptical $(T \leq-4)$ galaxies. Blue and green squares show those blue and red star-forming isolated galaxies of the previous figures with halo mass estimates, respectively. The numbers are the ID in the UNAM-KIAS catalog for the blue SF galaxies. Magenta open squares correspond to the isolated RQEs. The dotted line in the left panel is a visual approximation for the trend of simulated elliptical galaxies in Niemi et al. (2010).

(see Table 1 for mean and median values). In general, in both environments the fractions of blue and SF ellipticals are low. However, these fractions are larger for the isolated ellipticals (approximately $20 \%$ and $8 \%$, respectively) than for the Coma supercluster (approximately $8 \%$ and $\lesssim 1 \%$, respectively), as seen in Figs. 2 and 3. Moreover, these fractions deviate in a different way from the main red/passive sequences as a function of mass. The main difference is that the blue/SF isolated ellipticals deviate more from the main trends toward smaller masses down to $\log \left(M_{\mathrm{s}} / h^{-2} M_{\odot}\right) \approx 9.85$ (at lower masses, there are no blue nor $\mathrm{SF}$ E galaxies). In the case of the small fraction of blue ellipticals in the Coma supercluster, they deviate from the main trend only moderately, and below $\log \left(M_{\mathrm{s}} / h^{-2} M_{\odot}\right) \approx 10.4$ there are no blue ellipticals. Thus, in a high-density environment, only a small fraction of intermediate-mass galaxies are slightly away from the red sequence today, so that they seem to have intermediate-age stellar populations. The situation is not too different for the very isolated ellipticals, although as mentioned above, the fraction of blue objects is larger and, especially at intermediate masses, some of them deviate significantly from the red sequence and are actually SF galaxies. An interesting question is why these very isolated galaxies remain blue and star forming after their morphological transformation: Is that because this transformation happened recently from gaseous mergers or because they accreted gas, suffering a rejuvenation process? We discuss this question in Sect. 5.

A main result to be discussed now is that E galaxies in the local Universe are mostly red and dead (passive), that is, even those that are very isolated. It seems that the mechanisms responsible for the morphological transformation of galaxies produce efficient quenching of star formation and depletion of the cold gas reservoir, both for isolated and cluster E galaxies, and environment is not the most effective (or only) mechanism of quenching, although it is expected to play some role (see below Sect. 4.1).

In more detail, as can be seen in Figs. 2 and 4, both isolated and supercluster ellipticals follow the overall trend that they are on average redder as they are more massive (luminous). Moreover, above $M_{\mathrm{s}} \approx 8 \times 10^{10} h^{-2} M_{\odot}$ there are no blue or SF ellipticals at all in both the supercluster and isolated samples, and the luminosity-weighted ages are older than $4 \mathrm{Gyr}$ for almost all of them. This confirms that massive E galaxies assembled their stellar populations early, remaining quenched since those epochs (cf. Thomas et al. 2005; Schawinski et al. 2009; Kuntschner et al. 2010; Thomas et al. 2010). As we go to lower masses, notwithstanding the environment, ellipticals tend to have on average bluer colors than the more massive E galaxies. This mass downsizing behavior for E galaxies is also seen in the case of halo mass. The left panel of Fig. 9 shows the $B-R$ color as a function of $M_{\mathrm{h}}$ for the isolated E galaxies with an estimate of their halo (group) mass according to Y07. With a large scatter, we find that $B-R \propto 0.12 \log M_{\mathrm{h}}$. This trend, within the context of the merger-driven (morphological) quenching mechanism, might be explained by more gaseous and/or later mergers as the system is less massive; actually, observations show that lower mass galaxies have on average higher gas fractions (e.g., Avila-Reese et al. 2008; Papastergis et al. 2012; Calette et al. 2016; Lehnert et al. 2015). However, even if the merger is late and gaseous, the quenching seems to be so efficient and rapid that most of the low-mass ellipticals, both isolated and in the Coma supercluster, already transited to the red sequence by $z \sim 0$. Even more, their present-day sSFR's are very low, close to those of the massive galaxies or haloes (see Fig. 3 and right panel of Fig. 9).

Is it possible to evaluate in a more quantitative way whether the quenching of those E galaxies that are passive today happened early or recently? The very red colors of most massive ellipticals $\left(M_{\mathrm{S}} \gtrsim 10^{11} h^{-2} M_{\odot}\right)$ suggest that these galaxies did not have active star formation since at least 1-2 Gyr ago. However, those (less massive) passive E galaxies with bluer colors and sSFRs slightly higher than the average could have been quenched more recently. As described in Sect. 3.3, in the plot presented in Fig. 5, we can select those RQE galaxies. At face value, our results show that the ellipticals in an environment such as the 
Coma supercluster suffered the quenching of star formation, and likely the previous morphological transformation, early, so that at $z \sim 0$ almost all of them are passive and red; the small fraction that lies below the red sequence $(\approx 8 \%$ in Fig. 2 , likely in the green valley) are not RQEs excepting one marginal case, i.e. these ellipticals started to be quenched long ago but they are just in the process of transition to the red sequence.

In the case of isolated ellipticals, among those that are blue but passive galaxies ( 15\%), half are RQEs (see Figs. 2 and 5). The fact that in the isolated environment, among the blue passive ellipticals there is a significantly larger fraction of RQEs than in the Coma supercluster, suggests that the transition to the red sequence should be faster for isolated ellipticals than for supercluster ones. For the blue passive isolated E galaxies, we can even see a trend. The RQEs are bluer than those that were quenched not recently; most of the latter already lie very close to the red sequence (see Fig. 2). Instead, almost all of the (few) blue Coma ellipticals were quenched not recently but they did not yet arrive to the red sequence. Moreover, for masses below $\log \left(M_{\mathrm{s}} / h^{-2} M_{\odot}\right) \sim 10.5$, the isolated RQEs are close or already in the red sequence (excepting the peculiar case of the bluest galaxy to be discussed below; see Fig. 2). Therefore, they quenched and reddened very fast. In general, we see that as less massive the isolated RQEs are, the faster they seem to have been reddened. What produce the cessation of star formation and gas accretion so efficiently in isolated low-mass E galaxies?

\subsection{Quenching mechanisms of E galaxies}

Our results are consistent with the conclusions by Schawinski et al. (2014) for early-type galaxies in general. These authors propose that a major merger could simultaneously transform the galaxy morphology from disk to spheroid and cause rapid depletion of the cold gas reservoir with a consequent quenching of star formation (morphological quenching). As a result of the drop in star formation, the galaxy moves out of the blue cloud, into the green valley, and to the red sequence as fast as stellar evolution allows. The authors estimate that the transition process in terms of galaxy color takes about $1 \mathrm{Gyr}$ for early-type galaxies; this time is much longer for late-type galaxies, i.e., they undergo a much more gradual decline in star formation. The rapidity of the gas reservoir destruction in E galaxies should be due to a very efficient star formation process and strong supernovaand/or AGN-driven feedback (winds, ionization, etc.).

According to our results, on the one hand, isolated and Coma supercluster E galaxies in general share the same loci in the color $-M_{\mathrm{s}}$, color-magnitude, and $\mathrm{sSFR}-M_{\mathrm{s}}$ diagrams, and show evidence of rapid transition to the red sequence after they were quenched, especially the low-mass isolated ellipticals. On the other hand, the radius-mass relation of supercluster and isolated E galaxies are roughly similar and follow roughly the relation determined for early-type galaxies from large samples (Fig. 7). Moreover, as is well known, ellipticals in general are more concentrated than late-type galaxies. This makes evident the presence of strong dissipative processes at the basis of the origin in most of these galaxies, which are happening in the same way both for the isolated and cluster environments. In conclusion, the processes of morphological transformation, quenching, and rapid transition to the red sequence of $E$ galaxies seem to be in general independent of environment, except for a small fraction of isolated ellipticals that significantly deviate from the main sequences of E galaxies.

From an empirical point of view, the quenching of star formation in general has been found to be associated with mass and/or environment, mainly when the galaxy is a satellite (e.g., Peng et al. 2010b). Two main mechanisms of quenching associated with mass were proposed: (1) the strong virial shock heating of the gas in massive haloes (e.g., White \& Frenk 1991; Dekel \& Birnboim 2006); and (2) the AGN-driven feedback acting in massive galaxies assembled by major mergers (e.g., Silk \& Rees 1998; Binney 2004). Since the first models and simulations, where these mechanisms were implemented, it was shown that they are gradually more efficient as the halo mass increases, starting from $M_{\mathrm{h}} \sim 10^{12} M_{\odot}$ (e.g., Granato et al. 2004; Springel et al. 2005a; Di Matteo et al. 2005; Croton et al. 2006; Bower et al. 2006; De Lucia et al. 2006; Lagos et al. 2008; Somerville et al. 2008). This mass corresponds roughly to $M_{\mathrm{s}} \gtrsim 1.5 \times 10^{10} h^{-2} M_{\odot}$. As seen in Figs. 2 and 3 , both isolated and Coma supercluster E galaxies more massive than this follow roughly the same correlations of color and SSFR with mass, that is most of them are red and dead, regardless of the environment. Therefore, for massive ellipticals, rather than the environment, the physical mechanisms that depend on halo mass seem to be responsible for keeping E galaxies quiescent (see also Dekel \& Birnboim 2006; Woo et al. 2013; Yang et al. 2013; Dutton et al. 2015 , and more references therein). What is the situation for the lower mass ellipticals?

As seen in Figs. 2 and 3, most of the E galaxies (isolated or from the Coma supercluster) with masses lower than $M_{\mathrm{s}}=$ $1.5 \times 10^{10} h^{-2} M_{\odot}$ are also red and passive, although for these E galaxies, the quenching mechanisms associated with mass are not already suitable. While the hostile environment of clusters contributes to removing and to not fostering new episodes of cold gas inflow in the E galaxies, this is not the case for the isolated ellipticals. In fact, it should be said that most of galaxies with masses lower than $M_{\mathrm{S}}=1-1.5 \times 10^{10} h^{-2} M_{\odot}$ in the local Universe are centrals, gas-rich, blue, and SF (see, e.g., Weinmann et al. 2006; Yang et al. 2009, 2012), but they are also of late type. The question for these galaxies is why they delayed their active star formation phase to a greater degree the less massive they are (this is referred to as downsizing in star formation rate; e.g., Fontanot et al. 2009; Firmani \& Avila-Reese 2010; Weinmann et al. 2012). In the (rare) cases that these low-mass galaxies suffer a morphological transformation to an elliptical, according to our results, they should also destroy the gas reservoirs and strongly quench star formation.

It is expected that a major merger induces an efficient process of gas exhaustion due to enhanced star formation, but if after the merger a fraction of gas is left and/or the galaxy further accretes gas, then it could form stars again and become blue, SF, and even have a new disk (for theoretical works see, e.g., Robertson et al. 2006; Governato et al. 2009; Hopkins et al. 2009; Tutukov et al. 2011; Kannan et al. 2015, and for observational evidence see, e.g., Kannappan et al. 2009; Hammer et al. 2009; Puech et al. 2012). These processes are very unlikely to happen in a group/cluster environment, as mentioned above, but according to our results, late gas accretion and star formation do not occur in most of the very isolated $\mathrm{E}$ galaxies either since they are mostly red $(80 \%)$ and passive $(92 \%)$, and among those that are blue (20\%), only less than one-fourth are SF.

The quenching associated with mass can be very efficient for isolated $\mathrm{E}$ galaxies formed in haloes much more massive than $10^{12} M_{\odot}$. In the case of ellipticals formed in less massive haloes, a possible mechanism for ejecting remaining gas or for avoiding further cold gas infall could be the feedback produced by type Ia supernovae ( $\mathrm{SNe}$ Ia), which are not associated with the current star formation rate (SFR; e.g., Ciotti et al. 1991; Pellegrini 2011). Because the interstellar medium of $E$ galaxies is very 
poor, the energy and momentum released by $\mathrm{SNe}$ Ia are easily expected to attain the intrahalo medium, heat it, and eventually eject it from the low-mass halo. Moreover, the smaller the halo, the more efficient the feedback-driven outflows are expected to be. According to Figs. 2 and 3, the lowest mass, isolated $\mathrm{E}$ galaxies are all red/passive, and there are pieces of evidence that the less massive the isolated elliptical, the faster it quenched and reddened. On the other hand, Peng et al. (2015) have suggested recently that the primary mechanism responsible for quenching star formation is strangulation (or starvation). In this process, the supply of cold gas to the galaxy is halted with a typical timescale of 4 Gyr for galaxies with stellar mass less than $10^{11} M_{\odot}$. However, it is not clear what could be the mechanisms behind strangulation in an isolated environment. A possibility to have in mind is the removal of gas in early-formed, low-mass haloes due to ram pressure as they fly across pancakes and filaments of the cosmic web (Benítez-Llambay et al. 2013).

\subsection{Comparisons with theoretical predictions}

Within the context of the $\Lambda \mathrm{CDM}$ cosmology, the hierarchical mass assembly of dark haloes happens by accretion and minor/major mergers (see, e.g., Fakhouri \& Ma 2010, and more references therein). The average mass accretion and minor/major merger rates depend on mass and environment (e.g., Maulbetsch et al. 2007; Fakhouri \& Ma 2009). As a function of environment, present-day haloes in high-density regions suffered more major mergers on average and assembled a larger fraction of their mass in mergers than haloes in low-density regions (Maulbetsch et al. 2007). The latter continue growing today mainly by mass accretion. Therefore, at face value, we expect that the very isolated galaxies could be efficiently accreting mass at present due to the cosmological mass accretion of their haloes.

The accretion and merger histories of CDM haloes is the first step to calculate the mass assembly and morphology of the galaxies formed inside them. In this line of reasoning, it could be expected that the isolated $\mathrm{E}$ galaxies formed in the $\Lambda \mathrm{CDM}$ cosmology should be on average significantly bluer and with higher SFRs than the "normal" E galaxies formed in highdensity environments because the haloes of the former continue accreting mass by today (see above). Nevertheless, the galaxyhalo connection is by far not direct as a result of the nonlinear dynamics of the infalling subhaloes and the complex physical processes of the baryons, as several semiempirical studies have shown (see, e.g., Stewart et al. 2009; Hopkins et al. 2010; Zavala et al. 2012; Avila-Reese et al. 2014). As the result, for instance, Zavala et al. (2012) have shown that despite the fact that in the $\Lambda \mathrm{CDM}$ scenario the halo-halo major merger rates are high, this does not imply a problem of galaxy-galaxy major merger rates that are too high with the consequent overabundance of bulgedominated galaxies.

Schawinski et al. (2009) have compared their volumelimited SDSS sample of early-type galaxies (complete to $M_{r}=$ $-20.7 \mathrm{mag}$, which is slightly below $M^{*}$ ) to the $\Lambda$ CDM-based semianalytical models (SAM) of Khochfar \& Burkert (2005) and Khochfar \& Silk (2006). They found that these SAMs predict a slightly (significantly) higher fraction of blue (SF) early-type galaxies than the observed sample. In another work, by means of numerical simulations, Kaviraj et al. (2009) have found that the expected frequency of minor merging activity at low redshift can be consistent with the observed low-level of recent star formation activity in some early-type galaxies (Kaviraj et al. 2007). However, the theoretical study that is closest to the analysis presented here is that by Niemi et al. (2010). These authors have used the SAM results of De Lucia \& Blaizot (2007) built up in the Millennium Simulation (Springel et al. 2005b), and applied some criteria to select isolated galaxies and to determine which galaxies are ellipticals. They find that $26 \%$ of the synthetic isolated $\mathrm{E}$ galaxies should exhibit colors bluer than $B-R=1.4$ in an absolute magnitude range of $-21.5<M_{R}<-20$. They call this population the blue faint isolated ellipticals. In the UNAMKIAS catalog we find that only three isolated E galaxies satisfy the previous criteria (see Fig. 4), which corresponds to $3.4 \%$ of our pure E galaxy sample ( $0 \%$ of ellipticals in Coma). These three isolated ellipticals are part of the four blue SF galaxies (blue squares in all the plots shown in Sect. 3).

Part of the large discrepancy in the fractions of predicted and observed blue, faint galaxies can arise as a result of the different isolation criteria and morphological definitions used in Hernández-Toledo et al. (2010) with respect to Niemi et al. (2010). The isolation criteria of the former consider neighbor galaxies as not relevant perturbers if they have a magnitude difference of $\Delta m_{r} \geq 2.5$ compared to an isolated galaxy candidate within a radial velocity difference $\Delta V<1000 \mathrm{~km} \mathrm{~s}^{-1}$, whereas in the latter the difference in magnitude is $\Delta m_{B} \geq 2.2$ inside a sphere of $500 h^{-1} \mathrm{kpc}$ and this condition is relaxed to $\Delta m_{B} \geq 0.7$ for spheres with radii between $500 h^{-1} \mathrm{kpc}$ and $1 h^{-1} \mathrm{Mpc}$. We note that the former uses the $r$-band whereas the latter uses the $B$-band. Regarding the morphological definitions, in our case, galaxies are identified as ellipticals based on a structural and morphological analysis. They are denoted as $T \leq-4$ according to Buta et al. (1994). In the case of Niemi et al. (2010), they use the condition $T<-2.5$ to classify modeled galaxies as ellipticals, where the $T$ parameter is based on the $B$-band bulgeto-disk ratio. Therefore, these authors may have included some S0 galaxies in their sample. While these differences in the isolation criteria and morphological definitions could reduce the difference between our observed sample and the SAM prediction of Niemi et al. (2010), they are unlikely to explain the disagreement by a factor of around eight in the fractions of blue, faint isolated ellipticals.

It is known that in models and simulations the star formation of galaxies is to some point correlated with the dark matter accretion of their host haloes (e.g., Weinmann et al. 2012; González-Samaniego et al. 2014; Rodríguez-Puebla et al. 2016). The fact that a substantial population of the predicted blue, faint isolated galaxies is not observed suggests that the star formation activity of isolated E galaxies in the last Gyr(s) is overestimated in the SAMs. The overestimate in the star formation activity is likely due to the late (dark and baryonic) highmass accretion rate typical of isolated haloes (see discussion above). Indeed, Niemi et al. (2010) report that haloes hosting model isolated ellipticals continue their dark matter accretion until $z \sim 0$, whereas haloes hosting normal (nonisolated) ellipticals have joined nearly all their mass at $z \sim 0.5$. Furthermore, isolated ellipticals with halo masses $<10^{12} h^{-1} M_{\odot}$ have half of their stellar mass by $z \sim 0.7$, whereas isolated elliptical galaxies hosted by more massive haloes have half of their stellar mass by $z \sim 1.6$. Therefore, the different mass assembly histories of the SAM isolated ellipticals hosted by low-mass haloes explain their bluer colors compared to normal model ellipticals and other more massive isolated ellipticals.

Thus, the large difference we find here in the fraction of blue faint isolated $\mathrm{E}$ between observations and the SAMs, strongly suggest that some gastrophysical processes are yet missed in the SAMs, in particular at low masses. We have proposed the SN Iadriven feedback could be a mechanism that is able to avoid a significant population of blue faint ellipticals in isolated low-mass 
Table 2. General properties of the blue SF isolated elliptical galaxies.

\begin{tabular}{lccccccccc}
\hline \hline Name & $z$ & $\log \left(M_{\mathrm{s}}\right)$ & $g-i$ & $\log (\mathrm{s} S F R)$ & ${ }^{0.0} M_{\mathrm{R}}$ & $B-R$ & $R_{\mathrm{deV}}$ & $\operatorname{Age}$ & $\log \left(M_{\mathrm{h}}\right)$ \\
\hline UNAM-KIAS 359 & 0.017 & 9.86 & 0.94 & -10.26 & -20.50 & 1.38 & 1.50 & 868.0 & $\ldots$ \\
UNAM-KIAS 613 & 0.027 & 10.30 & 1.04 & -10.25 & -21.23 & 1.48 & 1.53 & 372.5 & 11.93 \\
UNAM-KIAS 1197 & 0.032 & 10.15 & 0.77 & -10.50 & -21.38 & 1.21 & 2.13 & 546.8 & 11.78 \\
UNAM-KIAS 1394 & 0.035 & 10.13 & 0.81 & -9.63 & -21.34 & 1.25 & 1.01 & 268.2 & 11.75 \\
\hline
\end{tabular}

Notes. Columns are: name in the UNAM-KIAS catalog, redshift, $\log _{10}$ of the stellar mass in units of $h^{-2} M_{\odot}, g-i$ color, $\log _{10}$ of the specific star formation rate in units of $\mathrm{yr}^{-1}$, absolute magnitude in the $R$-band $(h=0.7), B-R$ color, radius of a de Vaucouleurs fit in the $r$-band in units of kpc $(h=0.7)$, luminosity-weighted stellar age in units of Myr from the STARLIGHT database, and, if it is available, $\log _{10}$ of the host halo mass from Y07 in units of $h^{-1} M_{\odot}$.

haloes. In fact, the SAMs by De Lucia \& Blaizot (2007) included the effects of the SN Ia feedback, but in a very simple (parametric) way. More detailed studies of this process and of the mentioned above "cosmic web stripping", which affects low-mass haloes (Benítez-Llambay et al. 2013), are necessary.

In spite of the differences in the fractions of blue and faint isolated ellipticals between SAMs and observations, it should be said that the SAM predictions for the overall population of isolated $\mathrm{E}$ galaxies are consistent in general with our observed sample. The mean $B-R$ color for the synthetic isolated $\mathrm{E}$ galaxies in Niemi et al. (2010) is $1.47 \pm 0.23$, which is bluer than the mean for our observed isolated ellipticals $(1.62 \pm 0.10$, see Table 1), but yet within the scatters. On the other hand, the mean $B-R$ color for their modeled normal (nonisolated) E galaxies is $1.58 \pm 0.10$, which is bluer than the mean of E galaxies in the Coma supercluster $(1.64 \pm 0.05)$, but within the scatters again. These results show also that for both observations and models, the $B-R$ color distribution of $\mathrm{E}$ galaxies in isolated environments is broader than those in environments of higher density.

The linear fits of Niemi et al. (2010) to their normal and isolated ellipticals are shown in the CMD of Fig. 4 (dotted and solid lines, respectively). As can be seen, the ellipticals in the Coma supercluster follow the trend predicted by the model normal elliptical galaxies, but the same behavior is observed for many of our isolated ellipticals. Reda et al. (2004) found a similar result using a small observational sample of six bright isolated elliptical galaxies compared to the Coma cluster. However, there is a small fraction of isolated $\mathrm{E}$ galaxies that detach from this trend, toward bluer colors at fainter magnitudes. This trend is similar to that followed in the SAM by the isolated ellipticals, while, as reported above, the fraction of galaxies following this trend is much higher in the SAM than in observations. In the $(B-R)-M_{\mathrm{h}}$ diagram (left panel of Fig. 9), we plot with a dotted line a visual approximation for the trend of the model nonisolated ellipticals in Niemi et al. (2010). These authors predict that normal ellipticals have a roughly constant color for the whole halo mass range sampled $\left(10^{10}<M_{h} / h^{-1} M_{\odot}<10^{14}\right)$, but isolated ellipticals show bluer colors at $M_{h}<10^{12} h^{-1} M_{\odot}$. This behavior is somewhat similar for our observed isolated $\mathrm{E}$ galaxies, thus suggesting that some isolated ellipticals hosted by low/intermediummass haloes should exhibit bluer colors than normal ellipticals in haloes with the same mass.

We conclude that the $\Lambda$ CDM-based models of galaxy evolution are roughly consistent with observations in regards to the local population of E galaxies, both isolated and in cluster-like environments. However, the SAMs predict a too high abundance of blue, faint isolated ellipticals formed in low-mass haloes with respect to our observational inference. The above discussed mechanisms (SN-Ia feedback and cosmic web stripping) could help to avoid further gas accretion to galaxies in low/intermedium-mass haloes.

\section{The blue SF isolated ellipticals}

In the previous sections, we have shown that isolated E galaxies, as well as those in the Coma supercluster, are mostly red and passive. However, in the isolated environment, there is a small fraction of ellipticals that systematically deviate from the $\mathrm{red} /$ passive sequence as their masses are smaller. The question is whether these few intermedium-mass ellipticals are blue and SF because they suffered the morphological transition recently and did not yet exhaust their gas reservoir (McIntosh et al. 2014; Haines et al. 2015), which can include the process of disk regeneration as suggested by Kannappan et al. (2009), or because these ellipticals were rejuvenated by recent events of cold gas accretion as suggested by Thomas et al. (2010).

In general, several pieces of evidence show that the structural properties and correlations of blue SF isolated ellipticals do not differ significantly from those of the other isolated ellipticals or even the group/cluster ellipticals. For example, in Fig. 7 we show that the radius $-M_{\mathrm{s}}$ correlation of all ellipticals is roughly the same. Kannappan et al. (2009) found that blue-sequence E/S0s are more similar to red-sequence E/SOs than to late-type galaxies in the $M_{\mathrm{s}}$-radius relation. Blue $\mathrm{E} / \mathrm{S} 0$ galaxies are also closer to red $\mathrm{E} / \mathrm{S} 0$ than to late-type systems in this relation at $0.2<z<1.4$ (Huertas-Company et al. 2010). We need to go in more detail and explore whether the blue SF isolated ellipticals have some peculiarities that could suggest us which mechanisms are dominant in making them blue and SF.

A characterization of first order on the nature of the blue $\mathrm{SF}$ isolated $\mathrm{E}$ galaxies is proposed here using the available gri images and spectra from the SDSS database. This includes only four galaxies, three of which coincide with the definition of blue, faint isolated ellipticals in Niemi et al. (2010). Table 2 summarizes the general properties of these galaxies. In Apprendix A, we present an analysis of several structural-morphological and spectroscopical properties for each one of the four observed blue SF isolated ellipticals. We point out that UNAM-KIAS 1197 shows evidence that it is classified as a LINER galaxy and that UNAM-KIAS 613 could be actually an AGN due to its broad component in $\mathrm{H} \alpha$ (see Appendix A for details). We have also carried out similar analyses for the other, more common red and passive isolated ellipticals (to be presented elsewhere). In the following, we discuss the results of our analysis with the aim of elucidating the nature of the blue SF isolated ellipticals.

From the surface brightness profiles in the bands $g$ and $i$, we find that the four blue SF isolated ellipticals show radial color gradients with bluer colors toward the galaxy center (see bottomleft panels in Figs. A.1, A.2, A.4 and A.5 in Appendix A), while most of the red and passive ellipticals show negative or flat radial color profiles (e.g., den Brok et al. 2011). The positive color gradient may be evidence of dissipative infall of cold gas, which 
promotes recent star formation in the central regions. This supports the rejuvenation scenario for the blue SF ellipticals. Suh et al. (2010) have suggested that positive color gradients in earlytype galaxies are visible only for $0.5-1.3$ billion years after a star formation event. Afterward, the galaxies exhibit negative color gradients. Shapiro et al. (2010) have also proposed the rejuvenated scenario for some early-type galaxies in the SAURON sample (de Zeeuw et al. 2002) with red optical colors that show star formation activity in the infrared. These galaxies correspond to fast-rotating systems with concentrated star formation. They suggest that when the star formation ceases, over the course of $\sim 1 \mathrm{Gyr}$, the transiently star-forming galaxy return to evolve passively. Furthermore, Young et al. (2014) have also proposed the rejuvenation scenario to explain the blue tail of early-type galaxies in the color-magnitude diagram in the ATLAS ${ }^{3 \mathrm{D}}$ sample (Cappellari et al. 2011).

From our detailed photometric analysis (Appendix B), we find that the structure of isolated $\mathrm{E}$ galaxies can be described in general by three Sérsic components (inner, intermediate, and outer), which is usually reported for ellipticals in groups and clusters (e.g., Huang et al. 2013b). However, the outer components of our blue SF isolated ellipticals (see top-right panels of Figs. A.1 A.2, A.4 and A.5 and Table A.1 in Appendix A) are present in two cases, UNAM-KIAS 613 and UNAM-KIAS 1197 , where the latter shows a small value of $n$, and in other two cases it seems that this component is even absent, UNAMKIAS 359 and UNAM-KIAS 1394. On the contrary, bright cluster galaxies have been reported to have extended stellar envelopes with large $n$ indexes (Morgan \& Lesh 1965; Oemler 1974; Schombert 1986). For a sample of low-luminosity ellipticals, most of them in group-like environments, Huang et al. (2013b) measured a mean $n$ value of $1.6 \pm 0.5$ and a mean effective radius $r_{\text {eff }}=7.4 \pm 2.6 \mathrm{kpc}$ for the outer component. Only UNAM-KIAS 613 has a $n$ value larger than this mean and all the blue SF isolated ellipticals have values of the outer $r_{\text {eff }}$ that are smaller than the mentioned mean. Therefore, at least three of the four blue SF isolated ellipticals seem to have an outer structure that is different from other ellipticals.

According to Huang et al. (2013a), the different structural components in E galaxies may be explained by a two-phase scenario (Oser et al. 2010; Johansson et al. 2012). The inner and/or intermediate components are the outcomes of an initial phase characterized by dissipative (in situ) processes such as cold accretion or early gas-rich mergers. The outer, extended component of ellipticals are related to a second phase dominated by nondissipative (ex situ) processes such as dry, minor mergers after the quenching of the galaxy. This could also explain the build up of E galaxies in isolated environments. Galaxies with very small outer $n$ and $r_{\text {eff }}$ values or with no outer component, which is the case in three out of four blue SF isolated ellipticals, could have not suffered ex situ processes recently. This again supports the rejuvenation scenario for the blue SF ellipticals, at least for three of them.

Regarding the inner components of the blue SF isolated ellipticals, the results of our study show that their best-fit Sérsic indices have $n \leq 2.0$ and $r_{\text {eff }} \leq 0.6 \mathrm{kpc}$ (see Table A.1 in Appendix A), whereas Huang et al. (2013b) obtained mean values of $n=3.2 \pm 2.1$ and $r_{\text {eff }}=0.7 \pm 0.4 \mathrm{kpc}$ for their sample of ellipticals. Thus, though within the scatter, the blue SF isolated ellipticals seem to have a more disky inner component than other low-luminosity ellipticals. This can be consistent with both the rejuvenation by cold gas infall or the post-merger disk regeneration scenarios.
In Appendix B.1, we list the different fine structure and residual features that can be found in the images of E galaxies and their association with different levels of interaction/merger. Our analysis (see Table A.1) shows that two of the blue SF isolated ellipticals (UNAM-KIAS 613 and UNAM-KIAS 1394) do not present convincing evidence of (recent) disturbances of any type and the other two, UNAM-KIAS 1197 and UNAM-KIAS 359, present evidence of weak disturbance effects through low surface brightness (LSB) outer shells. We found no evidence of tidal tails or broad fans of stellar light, which are both associated with dynamically cold components produced by an accreted major companion. No evidence of significant sloshing (>10\%) in the inner kpc region was found either. An additional revision of the residuals in the very central regions suggests the presence of a few thin localized patches, which we tentatively interpret as coming from dusty features. This is consistent with the reddened central subregions observed in the corresponding color maps. Such presumably nuclear dust structures may be associated with the inner or intermediate disk-like components found in our decomposition analysis, and could be evidence of centralized star formation due to cold gas infall. Thus, the lack of evident fine structure and residual features and the presence of nuclear dust structures in the four blue SF isolated ellipticals supports again the mechanism of rejuvenation in contraposition to the one of recent mergers and disk regeneration. We caution, as noted by George \& Zingade (2015), that the SDSS images are not the most adequate for detecting finer details in early-type galaxies. Deeper imaging data is preferable.

Finally, in the Appendix $\mathrm{C}$ we describe our spectroscopic analysis for the four blue SF isolated elliptical galaxies, where the SDSS spectra were used. Recall that SDSS provides only one optical fiber of 3 arcsec aperture centered in each galaxy. For our four objects, this corresponds to the inner $\approx 1-2 \mathrm{kpc}$, which roughly corresponds to their de Vacouleours effective radii (Fig. 7). Our analysis of the emission lines gives similar results in the BPT diagram as those reported in the STARLIGHT database and plotted in Fig. 8 above (the loci of our estimates are indicated with a blue open square). We note that UNAM-KIAS 613 has very broad components in $\mathrm{H} \alpha$ and a clear pseudocontinuum (see Figs. A.2 and A.3 in Appendix A). UNAM-KIAS 1197 qualifies as a LINER, UNAM-KIAS 359 as a TO, and only UNAM-KIAS 1394 shows a clear evidence of dominant central star formation. Our analysis suggests then that the ionization mechanism could have a large contribution from a nuclear nonthermal component (e.g., LINER) in two of the four blue SF isolated ellipticals. Thus, the star formation rate in these galaxies could be lower than that calculated from the $\mathrm{H} \alpha$ flux.

The stellar populations encode the mass assembly history over the lifetime of the blue SF isolated elliptical galaxies, which is important to gain insights on their formation and evolution. By applying a stellar population synthesis analysis (see Appendix C), the obtained mass-weighted star formation histories show that the four blue SF ellipticals formed $<5 \%(<20 \%)$ out of their present-day stellar masses in the last 1 (3) Gyrs (see Fig. C.1 and Table A.2). The obtained light-weighted star formation histories show that $30-60 \%$ of the present-day luminosity is due to star formation in the last $1 \mathrm{Gyr}$. These results suggest that the blue SF isolated ellipticals formed most of their stars early, but in the last $\sim 1$ Gyr they had a period of enhanced star formation. This enhanced period of star formation is reflected in the very small luminosity-weighted average ages obtained for these galaxies with respect to the rest of the ellipticals (see Fig. 6).

We can also estimate the star formation timescale (SFTS; Plauchu-Frayn et al. 2012) activity in each galaxy by calculating 
the difference of mass-weighted and light-weighted average stellar ages (age $\mathrm{e}_{m w}$ and age $_{l w}$, respectively) as $\Delta($ age $)=$ $10^{\log \left(\operatorname{age}_{\mathrm{mw}}\right)}-10^{\log \left(\mathrm{age}_{\mathrm{lw}}\right)}$. These values are reported in Table A.2. The SFTS is an indicator of how fast a galaxy created its stellar population, or how long the stellar activity was prolonged in this galaxy. A typical elliptical galaxy, for example, where star formation has stopped long ago, would be expected to have a short SFTS. The blue SF isolated E galaxies have SFTS values of $8.5 \mathrm{Gyr}$ on average. As a comparison, Plauchu-Frayn et al. (2012) find that early-type galaxies in Hickson compact groups (Hickson 1982; Bitsakis et al. 2010, 2015) have SFTS values of $3.3 \mathrm{Gyr}$, whereas similar isolated galaxies have values of $5.4 \mathrm{Gyr}$, meaning that the former have formed their stars over shorter timescales than isolated early-type galaxies. The blue SF isolated galaxies show higher values than those respective samples of early-type galaxies, which suggests a more prolongated star formation activity in these galaxies.

Our photometric and spectroscopic analyses of the rare four blue SF isolated ellipticals are not conclusive. However, our results suggest that in general these galaxies do not present evidence of strong recent disturbances or mergers in their structure and morphology but they have been forming stars since the last $\sim 1$ Gyr in the central regions; in two cases there is also some evidence of AGNs. We conclude that it is more plausible that these isolated E galaxies assembled early as other ellipticals but they were rejuvenated by recent $(<1 \mathrm{Gyr})$ accretion events of cold gas. On the other hand, integral field spectroscopy (IFS) has allowed the study of kinematic and stellar population properties of early-type galaxies. For example, in the SAURON (Bacon et al. 2001; Kuntschner et al. 2010; Shapiro et al. 2010), ATLAS $^{3 D}$ (Cappellari et al. 2011; Young et al. 2014; McDermid et al. 2015), and CALIFA (Sánchez et al. 2012; González Delgado et al. 2014, 2015) projects. Future observations with IFS will be crucial to shed more light on the formation and evolution of blue isolated elliptical galaxies.

In the Introduction we stated the possibility of using pure E galaxies in very isolated environments as "sensors" of gas cooling from the intergalactic medium. This cool gas, once trapped by a galaxy, should form stars. The fact that only a negligible fraction $(\$ 4 \%)$ of our sample of local isolated ellipticals are blue and SF suggests that the process of cooling and infall of gas from the warm-hot intergalactic medium is very inefficient.

\section{Conclusions}

We have studied a sample of 89 local very isolated $\mathrm{E}$ galaxies ( $z=0.037$ on average) and compared their properties with those E galaxies located in a higher density environment, the Coma supercluster. The samples studied here refer only to morphologically, well-defined elliptical (pure-spheroid) galaxies in the mass range $6 \times 10^{8} \lesssim M_{\mathrm{s}} / h^{-2} M_{\odot} \lesssim 2 \times 10^{11}$, in contrast to other works that select overall early-type galaxies including S0s objects. Our main results and conclusions are as follow.

(i) The correlations of color, sSFR, and size with mass that follow most of the isolated $\mathrm{E}$ galaxies are similar to those of the Coma supercluster E galaxies. Notwithstanding the environment, most of ellipticals are "red and dead". All E galaxies more massive than $M_{\mathrm{S}} \approx 5 \times 10^{10} h^{-2} M_{\odot}\left(M_{\mathrm{h}} \approx 10^{13} h^{-1} M_{\odot}\right)$ are quiescent. As the mass or luminosity is smaller, both isolated and Coma ellipticals become bluer, although on average they remain in the red sequence. However, a few intermediate-mass ellipticals pass to be moderately blue in Coma, while in the case of the isolated ellipticals, a fraction of them deviates systematically toward the blue cloud. The extreme of this branch is traced by those isolated ellipticals that are blue and SF at the same time; this includes only four ellipticals, which have intermediate stellar masses between $7 \times 10^{9}$ and $2 \times 10^{10} h^{-2} M_{\odot}$. These blue SF isolated ellipticals are also the youngest galaxies with lightweighted stellar ages $\lesssim 1 \mathrm{Gyr}$, which could indicate recent processes of star formation in them.

(ii) In terms of fractions, among the isolated ellipticals $\approx 20 \%$ are blue, $\$ 7 \%$ are $\mathrm{SF}$, and $\approx 4.5 \%$ are blue SF, while among the Coma ellipticals $\approx 8 \%$ are blue, $\lesssim 1 \%$ are $\mathrm{SF}$, and there are no blue SF objects. On average, the galaxies in Coma have sSFR values that are lower than isolated ellipticals by $\sim 0.2$ dex and are older by $\lesssim 1$ Gyr. Based on a color-color criterion, $\approx 10 \%$ of the isolated ellipticals show evidence of recent quenching. All of these isolated RQEs are less massive than $M_{\mathrm{s}} \approx 7 \times 10^{10} h^{-2} M_{\odot}$, and are approaching the red sequence (the two lowest massive ellipticals are actually already red), which suggests that the quenching and reddening happened quickly in the isolated environment. In the Coma supercluster, excepting one marginal case, there are no RQEs, even among those that are still blue; for the latter, the quenching and reddening seem to have proceeded more gradually.

(iii) Around $40 \%$ of the E galaxies have detectable $(S / N>$ 3 ) emission lines in both isolated and dense environments. According to the BPT diagram, most of these are AGNs. However, the fraction of those classified as SFN/TO is slightly larger for the isolated ellipticals than for those in the Coma supercluster (11\% and $8 \%$, respectively).

Our results show that all massive ellipticals $\left(M_{\mathrm{s}} \gtrsim 5 \times\right.$ $10^{10} h^{-2} M_{\odot}$ ), and a large fraction of the less massive ellipticals, assembled their stellar populations early, remaining quenched since these epochs, regardless of whether they are isolated or from the Coma supercluster. Moreover, in both of these different environments, a downsizing trend is observed: as the mass becomes lower, the ellipticals are on average less red and have higher sSFR. Thus, rather than environment, it seems that the processes involved in the morphological transformation of E galaxies are those that dominate in their efficient star formation shut-off, the depletion of their cold gas reservoir, and their downsizing trends. On the other hand, new episodes of cold gas inflow are very unlikely to happen in the environment of clusters or for isolated galaxies living in massive haloes, hence, the E galaxies are expected to remain quenched. However, our study shows that most of intermediate- and low-mass isolated ellipticals have also transited to the red/passive sequence by $z \sim 0$. We suggested two possible mechanisms to explain why most lowmass E galaxies in an isolated environment could be devoid of gas: (1) the galactic winds produced by the feedback of SNe Ia; and (2) the removal of gas in low-mass haloes due to ram pressure as they fly across pancakes and filaments of the cosmic web.

Interesting enough, the predictions of $\Lambda \mathrm{CDM}$-based SAMs for the population of $\mathrm{E}$ galaxies (both in clusters/groups and isolated) agree in general with the results of our study, except that these models predict a too high abundance (a factor of 8 more) of blue, faint (low-mass) isolated ellipticals with respect to our results. This suggests that some gastrophysical processes at low masses, for example, those mentioned above, are yet missed or underestimated in SAMs.

Our results show that $\mathrm{E}$ galaxies in the isolated environment are not too different from those in the Coma supercluster at a given mass, but the fractions of blue or SF objects is larger in the former case than in the latter. Hence, in some cases, the isolated environment seems to propitiate the rejuvenation or a late formation of the ellipticals. The extreme examples are those ellipticals that are blue and SF at the same time; they exist only in 
the isolated environment. In Appendix A, we presented a structural/spectroscopic analysis of these four ellipticals with the aim of inquiring about their nature. We found the following for them:

(iv) The four blue SF isolated ellipticals have radial color gradients with bluer colors toward the galaxy center. Furthermore, at least three out of the four blue SF isolated ellipticals have only two (inner/intermediate) structural components, lacking the third outer component seen in classical ellipticals. The four ellipticals lack significant fine structure and residual features, and show the presence of nuclear dust structures.

(v) The spectroscopic analysis suggests that the ionization mechanism can have a large contribution from a nuclear nonthermal component (e.g., LINER) in two of the four blue SF isolated ellipticals. On the other hand, $30-60 \%$ of their presentday luminosity, but only $<5 \%$ of their present-day mass, is due to star formation in the last $1 \mathrm{Gyr}$. This suggests that these galaxies formed most of their stars early but in the last $\sim 1$ Gyr they had a period of enhanced star formation. Their high SFTS values suggest that they have formed their stars over prolongated timescales.

The positive color gradient in the four blue SF isolated ellipticals may be evidence of recent cold gas infall, which supports the rejuvenation scenario in contraposition to the scenario of a recent merger and/or disk regeneration. The presence of only inner and intermediate structural components, which are related to dissipative processes such as cold accretion or early gas-rich mergers, and the lack of outer component, which is related to nondissipative processes (e.g., dry mergers) after the quenching of the galaxy, suggest that these ellipticals did not suffer recent dry mergers but probably had cold gas accretion. This is supported by the lack of fine structure and residual features and the nuclear dust structures.

We conclude that it is more plausible that the blue SF isolated E galaxies assembled early as other ellipticals, but they were rejuvenated by recent $(<1 \mathrm{Gyr})$ accretion events of cold gas. Further work with powerful observational methods such as IFS is needed to investigate the kinematic and stellar population properties resolved in space of the blue SF isolated elliptical galaxies. These galaxies can be used to trace and estimate the fraction of recent gas cooling from the cosmic web.

Acknowledgements. We thank Mariana Cano-Díaz for her valuable comments and suggestions about the modeling of the UNAM-KIAS 613 spectrum. We acknowledge the anonymous referee for his/her constructive comments on our paper. HMHT and V.A.R. acknowledge CONACyT grant (Ciencia Básica) 167332 for partial support. HMHT and J.A.S. acknowledge DGAPA PAPIIT IN-112912 for financial support. A.D.O. acknowledges financial support from the Spanish Ministry for Economy and Competitiveness through grant AYA2013-42227-P and from Junta de Andalucía TIC114. This research has made use of the GOLDMine Database. This research has also made use of the NASA/IPAC Extragalactic Database (NED) which is operated by the Jet Propulsion Laboratory, California Institute of Technology, under contract with the National Aeronautics and Space Administration.

\section{References}

Adelman-McCarthy, J. K., Agüeros, M. A., Allam, S. S., et al. 2007, ApJS, 172, 634

Avila-Reese, V., Zavala, J., Firmani, C., \& Hernández-Toledo, H. M. 2008, AJ, 136,1340

Avila-Reese, V., Zavala, J., \& Lacerna, I. 2014, MNRAS, 441, 417

Bacon, R., Copin, Y., Monnet, G., et al. 2001, MNRAS, 326, 23

Baldry, I. K., Glazebrook, K., Brinkmann, J., et al. 2004, ApJ, 600, 681

Baldwin, J. A., Phillips, M. M., \& Terlevich, R. 1981, PASP, 93, 5

Bamford, S. P., Nichol, R. C., Baldry, I. K., et al. 2009, MNRAS, 393, 1324

Bell, E. F., McIntosh, D. H., Katz, N., \& Weinberg, M. D. 2003, ApJS, 149, 289
Bell, E. F., Wolf, C., Meisenheimer, K., et al. 2004, ApJ, 608, 752

Benítez-Llambay, A., Navarro, J. F., Abadi, M. G., et al. 2013, ApJ, 763, L41

Binney, J. 2004, MNRAS, 347, 1093

Bitsakis, T., Charmandaris, V., Le Floc'h, E., et al. 2010, A\&A, 517, A75

Bitsakis, T., Dultzin, D., Ciesla, L., et al. 2015, MNRAS, 450, 3114

Blanton, M. R., \& Moustakas, J. 2009, ARA\&A, 47, 159

Blanton, M. R., \& Roweis, S. 2007, AJ, 133, 734

Bower, R. G., Benson, A. J., Malbon, R., et al. 2006, MNRAS, 370, 645

Brinchmann, J., Charlot, S., White, S. D. M., et al. 2004, MNRAS, 351, 1151

Bruzual, G., \& Charlot, S. 2003, MNRAS, 344, 1000

Buta, R., Mitra, S., de Vaucouleurs, G., \& Corwin, Jr., H. G. 1994, AJ, 107, 118

Calette, R., Avila-Reese, V., Rodríguez-Puebla, A., \& Hernández-Toledo, H. M. 2016, Rev. Mex. Astron. Astrofis., submitted

Cappellari, M., Emsellem, E., Krajnović, D., et al. 2011, MNRAS, 413, 813

Cardelli, J. A., Clayton, G. C., \& Mathis, J. S. 1989, ApJ, 345, 245

Choi, Y.-Y., Park, C., \& Vogeley, M. S. 2007, ApJ, 658, 884

Cid Fernandes, R., Mateus, A., Sodré, L., Stasińska, G., \& Gomes, J. M. 2005, MNRAS, 358, 363

Ciotti, L., D'Ercole, A., Pellegrini, S., \& Renzini, A. 1991, ApJ, 376, 380

Colbert, J. W., Mulchaey, J. S., \& Zabludoff, A. I. 2001, AJ, 121, 808

Collobert, M., Sarzi, M., Davies, R. L., Kuntschner, H., \& Colless, M. 2006, MNRAS, 370, 1213

Cooper, A. P., Martínez-Delgado, D., Helly, J., et al. 2011, ApJ, 743, L21

Croton, D. J., Springel, V., White, S. D. M., et al. 2006, MNRAS, 365, 11

de Carvalho, R. R., \& Djorgovski, S. 1992, ApJ, 389, L49

De Lucia, G., \& Blaizot, J. 2007, MNRAS, 375, 2

De Lucia, G., Springel, V., White, S. D. M., Croton, D., \& Kauffmann, G. 2006, MNRAS, 366, 499

de Zeeuw, P. T., Bureau, M., Emsellem, E., et al. 2002, MNRAS, 329, 513

Dekel, A., \& Birnboim, Y. 2006, MNRAS, 368, 2

den Brok, M., Peletier, R. F., Valentijn, E. A., et al. 2011, MNRAS, 414, 3052

Denicoló, G., Terlevich, R., Terlevich, E., et al. 2005, MNRAS, 356, 1440

Di Matteo, T., Springel, V., \& Hernquist, L. 2005, Nature, 433, 604

Dressler, A. 1980, ApJ, 236, 351

Dupraz, C., \& Combes, F. 1986, A\&A, 166, 53

Dutton, A. A., Conroy, C., van den Bosch, F. C., et al. 2011, MNRAS, 416, 322

Dutton, A. A., Macciò, A. V., Stinson, G. S., et al. 2015, MNRAS, 453, 2447

Eracleous, M., Lewis, K. T., \& Flohic, H. M. L. G. 2009, New Astron. Rev., 53, 133

Fakhouri, O., \& Ma, C.-P. 2009, MNRAS, 394, 1825

Fakhouri, O., \& Ma, C.-P. 2010, MNRAS, 401, 2245

Fasano, G., Vanzella, E., Dressler, A., et al. 2012, MNRAS, 420, 926

Finkelman, I., Brosch, N., Funes, J. G., Kniazev, A. Y., \& Väisänen, P. 2010, MNRAS, 407, 2475

Firmani, C., \& Avila-Reese, V. 2010, ApJ, 723, 755

Fontanot, F., De Lucia, G., Monaco, P., Somerville, R. S., \& Santini, P. 2009, MNRAS, 397, 1776

Gavazzi, G., Boselli, A., Donati, A., Franzetti, P., \& Scodeggio, M. 2003, A\&A, 400,451

Gavazzi, G., Franzetti, P., \& Boselli, A. 2014, ArXiv e-prints [arXiv: 1401.8123]

Gallazzi, A., Charlot, S., Brinchmann, J., White, S. D. M., \& Tremonti, C. A. 2005, MNRAS, 362, 41

George, K., \& Zingade, K. 2015, A\&A, 583, A103

Gezari, S., Halpern, J. P., \& Eracleous, M. 2007, ApJS, 169, 167

González Delgado, R. M., Pérez, E., Cid Fernandes, R., et al. 2014, A\&A, 562, A47

González Delgado, R. M., García-Benito, R., Pérez, E., et al. 2015, A\&A, 581, A103

González-Samaniego, A., Colín, P., Avila-Reese, V., Rodríguez-Puebla, A., \& Valenzuela, O. 2014, ApJ, 785, 58

Governato, F., Brook, C. B., Brooks, A. M., et al. 2009, MNRAS, 398, 312

Granato, G. L., De Zotti, G., Silva, L., Bressan, A., \& Danese, L. 2004, ApJ, 600,580

Guo, Y., McIntosh, D. H., Mo, H. J., et al. 2009, MNRAS, 398, 1129

Haines, T., McIntosh, D. H., Sánchez, S. F., Tremonti, C., \& Rudnick, G. 2015, MNRAS, 451, 433

Hammer, F., Flores, H., Puech, M., et al. 2009, A\&A, 507, 1313

Hau, G. K. T., \& Forbes, D. A. 2006, MNRAS, 371, 633

Hernández-Toledo, H. M., Vázquez-Mata, J. A., Martínez-Vázquez, L. A., Choi, Y.-Y., \& Park, C. 2010, AJ, 139, 2525

Hernquist, L. 1993, ApJ, 409, 548

Hickson, P. 1982, ApJ, 255, 382

Holden, B. P., van der Wel, A., Rix, H.-W., \& Franx, M. 2012, ApJ, 749, 96

Hopkins, P. F., Cox, T. J., Younger, J. D., \& Hernquist, L. 2009, ApJ, 691, 1168

Hopkins, P. F., Bundy, K., Croton, D., et al. 2010, ApJ, 715, 202

Huang, S., Ho, L. C., Peng, C. Y., Li, Z.-Y., \& Barth, A. J. 2013a, ApJ, 768, L28

Huang, S., Ho, L. C., Peng, C. Y., Li, Z.-Y., \& Barth, A. J. 2013b, ApJ, 766, 47 
Hubble, E. P. 1926, ApJ, 64, 321

Huertas-Company, M., Aguerri, J. A. L., Tresse, L., et al. 2010, A\&A, 515, A3

Ivezić, Ž., Connolly, A., Vanderplas, J. T., \& Gray, A. 2014, Statistics, Data

Mining and Machine Learning in Astronomy (Princeton University Press)

Johansson, P. H., Naab, T., \& Ostriker, J. P. 2012, ApJ, 754, 115

Kannan, R., Macciò, A. V., Fontanot, F., et al. 2015, MNRAS, 452, 4347

Kannappan, S. J., Guie, J. M., \& Baker, A. J. 2009, AJ, 138, 579

Karachentseva, V. E. 1973, Astrofizicheskie Issledovaniia Izvestiya Spetsialńoj Astrofizicheskoj Observatorii, 8, 3

Kauffmann, G. 1996, MNRAS, 281, 487

Kauffmann, G., Heckman, T. M., Tremonti, C., et al. 2003, MNRAS, 346, 1055

Kaviraj, S., Schawinski, K., Devriendt, J. E. G., et al. 2007, ApJS, 173, 619

Kaviraj, S., Peirani, S., Khochfar, S., Silk, J., \& Kay, S. 2009, MNRAS, 394, 1713

Kewley, L. J., Dopita, M. A., Sutherland, R. S., Heisler, C. A., \& Trevena, J. 2001, ApJ, 556, 121

Khochfar, S., \& Burkert, A. 2005, MNRAS, 359, 1379

Khochfar, S., \& Silk, J. 2006, MNRAS, 370, 902

Kroupa, P. 2001, MNRAS, 322, 231

Kuntschner, H. 2000, MNRAS, 315, 184

Kuntschner, H., Emsellem, E., Bacon, R., et al. 2010, MNRAS, 408, 97

Lacerna, I., Rodríguez-Puebla, A., Avila-Reese, V., \& Hernández-Toledo, H. M. 2014, ApJ, 788, 29

Lagos, C. D. P., Cora, S. A., \& Padilla, N. D. 2008, MNRAS, 388, 587

Lane, R. R., Salinas, R., \& Richtler, T. 2013, A\&A, 549, A148

Lane, R. R., Salinas, R., \& Richtler, T. 2015, A\&A, 574, A93

Lehnert, M. D., van Driel, W., \& Minchin, R. 2015, A\&A, accepted [arXiv: 1510.01959]

Lintott, C., Schawinski, K., Bamford, S., et al. 2011, MNRAS, 410, 166

Marcum, P. M., Aars, C. E., \& Fanelli, M. N. 2004, AJ, 127, 3213

Maulbetsch, C., Avila-Reese, V., Colín, P., et al. 2007, ApJ, 654, 53

McDermid, R. M., Alatalo, K., Blitz, L., et al. 2015, MNRAS, 448, 3484

McIntosh, D. H., Wagner, C., Cooper, A., et al. 2014, MNRAS, 442, 533

Morgan, W. W., \& Lesh, J. R. 1965, ApJ, 142, 1364

Mosleh, M., Williams, R. J., \& Franx, M. 2013, ApJ, 777, 117

Nair, P. B., \& Abraham, R. G. 2010, ApJS, 186, 427

Niemi, S.-M., Heinämäki, P., Nurmi, P., \& Saar, E. 2010, MNRAS, 405, 477

Oemler, Jr., A. 1974, ApJ, 194, 1

Oser, L., Ostriker, J. P., Naab, T., Johansson, P. H., \& Burkert, A. 2010, ApJ, 725,2312

Osterbrock, D. E. 1989, Astrophysics of gaseous nebulae and active galactic nuclei (Mill Valley, California: University of Science Books), 422

Papastergis, E., Cattaneo, A., Huang, S., Giovanelli, R., \& Haynes, M. P. 2012, ApJ, 759, 138

Pellegrini, S. 2011, ApJ, 738, 57

Peng, C. Y., Ho, L. C., Impey, C. D., \& Rix, H.-W. 2002, AJ, 124, 266

Peng, C. Y., Ho, L. C., Impey, C. D., \& Rix, H.-W. 2010a, AJ, 139, 2097

Peng, Y.-J., Lilly, S. J., Kovač, K., et al. 2010b, ApJ, 721, 193

Peng, Y., Maiolino, R., \& Cochrane, R. 2015, Nature, 521, 192

Plauchu-Frayn, I., Del Olmo, A., Coziol, R., \& Torres-Papaqui, J. P. 2012, A\&A, 546, A48

Popović, L. Č., Shapovalova, A. I., Ilić, D., et al. 2014, A\&A, 572, A66

Puech, M., Hammer, F., Hopkins, P. F., et al. 2012, ApJ, 753, 128

Reda, F. M., Forbes, D. A., Beasley, M. A., O'Sullivan, E. J., \& Goudfrooij, P. 2004, MNRAS, 354, 851

Richtler, T., Salinas, R., Lane, R. R., Hilker, M., \& Schirmer, M. 2015, A\&A, 574, A21

Roberts, M. S., \& Haynes, M. P. 1994, ARA\&A, 32, 115

Robertson, B., Bullock, J. S., Cox, T. J., et al. 2006, ApJ, 645, 986
Rodríguez-Puebla, A., Primack, J. R., Behroozi, P., \& Faber, S. M. 2016, MNRAS, 455, 2592

Salinas, R., Alabi, A., Richtler, T., \& Lane, R. R. 2015, A\&A, 577, A59

Sánchez, S. F., Kennicutt, R. C., Gil de Paz, A., et al. 2012, A\&A, 538, A8

Schauer, A. T. P., Remus, R.-S., Burkert, A., \& Johansson, P. H. 2014, ApJ, 783, L32

Schawinski, K., Thomas, D., Sarzi, M., et al. 2007, MNRAS, 382, 1415

Schawinski, K., Lintott, C., Thomas, D., et al. 2009, MNRAS, 396, 818

Schawinski, K., Urry, C. M., Simmons, B. D., et al. 2014, MNRAS, 440, 889

Schlegel, D. J., Finkbeiner, D. P., \& Davis, M. 1998, ApJ, 500, 525

Schombert, J. M. 1986, ApJS, 60, 603

Shapiro, K. L., Falcón-Barroso, J., van de Ven, G., et al. 2010, MNRAS, 402, 2140

Shen, B. S., Mo, H. J., White, S. D. M., et al. 2007, MNRAS, 379, 400

Shen, S., Mo, H. J., White, S. D. M., et al. 2003, MNRAS, 343, 978

Silk, J., \& Rees, M. J. 1998, A\&A, 331, L1

Smith, R. M., Martínez, V. J., \& Graham, M. J. 2004, ApJ, 617, 1017

Smith, R. M., Fernández-Soto, A., Martínez, V., et al. 2010, in Galaxies in Isolation: Exploring Nature Versus Nurture, eds. L. Verdes-Montenegro, A. Del Olmo, \& J. Sulentic, ASP Conf. Ser., 421, 221

Somerville, R. S., Hopkins, P. F., Cox, T. J., Robertson, B. E., \& Hernquist, L. 2008, MNRAS, 391, 481

Springel, V., Di Matteo, T., \& Hernquist, L. 2005a, MNRAS, 361, 776

Springel, V., White, S. D. M., Jenkins, A., et al. 2005b, Nature, 435, 629

Stewart, K. R., Bullock, J. S., Wechsler, R. H., \& Maller, A. H. 2009, ApJ, 702, 307

Stocke, J. T., Keeney, B. A., Lewis, A. D., Epps, H. W., \& Schild, R. E. 2004, AJ, 127, 1336

Stoughton, C., Lupton, R. H., Bernardi, M., Blanton, M. R., et al. 2002, AJ, 123, 485

Strateva, I. V., Strauss, M. A., Hao, L., et al. 2003, AJ, 126, 1720

Suh, H., Jeong, H., Oh, K., et al. 2010, ApJS, 187, 374

Thomas, D., Maraston, C., Bender, R., \& Mendes de Oliveira, C. 2005, ApJ, 621,673

Thomas, D., Maraston, C., Schawinski, K., Sarzi, M., \& Silk, J. 2010, MNRAS, 404, 1775

Tutukov, A. V., Dryomov, V. V., \& Dryomova, G. N. 2007, Astron. Rep., 51, 435

Tutukov, A. V., Lazareva, G. G., \& Kulikov, I. M. 2011, Astron. Rep., 55, 770

van den Bosch, F. C., Aquino, D., Yang, X., et al. 2008, MNRAS, 387, 79

Veilleux, S., \& Osterbrock, D. E. 1987, ApJS, 63, 295

Véron-Cetty, M.-P., \& Véron, P. 2006, A\&A, 455, 773

Vulcani, B., Poggianti, B. M., Fritz, J., et al. 2015, ApJ, 798, 52

Wang, T.-G., Dong, X.-B., Zhang, X.-G., et al. 2005, ApJ, 625, L35

Weinmann, S. M., van den Bosch, F. C., Yang, X., \& Mo, H. J. 2006, MNRAS, 366,2

Weinmann, S. M., Pasquali, A., Oppenheimer, B. D., et al. 2012, MNRAS, 426, 2797

White, S. D. M., \& Frenk, C. S. 1991, ApJ, 379, 52

Yang, X., Mo, H. J., van den Bosch, F. C., et al. 2007, ApJ, 671, 153

Woo, J., Dekel, A., Faber, S. M., et al. 2013, MNRAS, 428, 3306

Yang, X., Mo, H. J., \& van den Bosch, F. C. 2008, ApJ, 676, 248

Yang, X., Mo, H. J., \& van den Bosch, F. C. 2009, ApJ, 695, 900

Yang, X., Mo, H. J., van den Bosch, F. C., Zhang, Y., \& Han, J. 2012, ApJ, 752, 41

Yang, X., Mo, H. J., van den Bosch, F. C., et al. 2013, ApJ, 770, 115

York, D. G., Adelman, J., Anderson, Jr., J. E., et al. 2000, AJ, 120, 1579

Young, L. M., Scott, N., Serra, P., et al. 2014, MNRAS, 444, 3408

Zavala, J., Avila-Reese, V., Firmani, C., \& Boylan-Kolchin, M. 2012, MNRAS, 427, 1503 


\section{Appendix A: Results for individual blue SF isolated elliptical galaxies}

Various image procedures were applied to the SDSS gri-band images to extract information about the internal and external structure of these galaxies. The details of the methodology are in Appendix B. On the other hand, a population synthesis model was applied to the SDSS spectra to extract information on the stellar populations and gas properties of their central regions. The methodology is explained in Appendix C. The results of each galaxy are summarized in Tables A.1 and A.2 and presented in Figs. A.1, A.2, A.4, and A.5 with the following set of panels:

(a) Top left. First row from left to right shows an gri image of the galaxy from the SDSS database, a sharp-filtered image with a $\sigma=4$ for the Gaussian function (in pixels) along the direction $\theta$ of the major axis of the Gaussian function and $g-i$ color index map. Second row from left to right shows the best set of Sérsic profiles for the observed surface brightness distribution in the $r$-band, which is summarized as the residual image after subtracting the best first model (Sérsic1), the best second model (Sérsic2), and the best third model (Sérsic3). Images are registered at the nuclear region of each galaxy. The individual components found in the 2D decomposition are roughly represented with ellipses in different colors (red, blue, green, and cyan); each ellipse shows the semimajor axis in units of $r_{\mathrm{eff}}$ using the $b / a$ axis ratio of each best Sérsic model.

(b) Top right. A comparison of azimuthally averaged 1D $r$-band profiles of the data and the best global model along with the corresponding residuals (lower box). Both the observed and best-model azimuthally averaged 1D profiles were extracted by imposing a fixed center and free ellipticity $\epsilon$ and position angle estimates. In general, the azimuthally averaged 1D surface brightness profiles built from this model solution reproduce the observed profiles reasonably well.

(c) Bottom left. Surface brightness profile in the $g$ and $i$ bands. The profiles were extracted by imposing a fixed center and fixed ellipticity $\epsilon$ and position angle estimates to guarantee homogeneous color estimates. The lower panel shows the $g-i$ color gradient, corrected for galactic extinction.

(d) Bottom right. The observed and modeled spectrum with STARLIGHT, and the residual spectrum (lower box).

In the following analysis, we use $h=0.73, \Omega_{\mathrm{M}}=0.27, \Omega_{\Lambda}=$ 0.73 along with a correction by Virgo centric infall to estimate the physical scales (e.g., $r_{\text {eff }}$ and fiber size). We here summarize the main features found from our analysis for each of the four blue SF isolated ellipticals. We explicitly repeated the spectroscopic analysis for the following four isolated ellipticals by applying the available STARLIGHT code, modeling their onefiber SDSS spectra, and then fitting the emission lines from the residual (observed - modeled) spectra.

\section{A.1. UNAM-KIAS 359}

No similar-sized neighbor galaxy is found within $727 \mathrm{kpc}$ and $\pm 1000 \mathrm{~km} \mathrm{~s}^{-1}$. The color map in the top-left panel of Fig. A.1 shows a conspicuous central region with a uniform reddened cusp but asymmetric reddening distribution, an intermediate bluer region, and an outer diffuse red region.

Our 2D residual images show some tiny central residuals consistent with the color index map showing a strong reddened central cusp and an adjacent reddened patch to the right, probably associated with dusty structures. There is marginal evidence of external low surface brightness features. This galaxy does not show any significant sloshing in the inner kpc region. This galaxy has been reported as detected in H I $21 \mathrm{~cm}$.

Our analysis from GALFIT shows three inner components $\left(r_{\mathrm{eff}}=0.15,0.55\right.$, and $\left.0.80 \mathrm{kpc}\right)$, all with Sérsic indices lower than 1 ( $n=0.1,0.15$, and 0.30 , respectively), and one intermediate-outer component $\left(r_{\text {eff }}=1.95 \mathrm{kpc}\right)$ with $n=1.00$.

At the distance of UNAM-KIAS 359, the 3 arcsec SDSS fiber spectrum subtends $1.04 \mathrm{kpc}$. From our spectroscopic analysis, this galaxy qualifies in the BPT diagram as a transition object (TO) with a mixture of a central, low-luminosity AGN and emission coming from a circumnuclear SF region (see Fig. 8).

\section{A.2. UNAM-KIAS 613}

Only one neighbor galaxy fainter than $2.5 \mathrm{mag}$ with respect to UNAM-KIAS 613 is within $712 \mathrm{kpc}$ and $\pm 1000 \mathrm{~km} \mathrm{~s}^{-1}$. The SDSS images in the top-left panel of Fig. A.2 show (i) a conspicuous nuclear region (maybe a lens) surrounded by (ii) an adjacent more diffuse region and also an (iii) outer envelope. The central strongly reddened cusp region contrasts with the more bluish colors of the rest of the galaxy in the color map.

The $2 \mathrm{D}$ residual images show a few weak filamentary features, probably associated with dust structures. The sloshing is not significant in the inner kpc. No low surface brightness features are observed in the outer regions of this galaxy. The galaxy was classified as S0 by Nair \& Abraham (2010).

Our analysis from GALFIT, summarized in the top-right panel of Fig. A.2, shows three components: an inner one $\left(r_{\mathrm{eff}}=\right.$ $0.43 \mathrm{kpc})$ with Sérsic index $n=1.47$, an intermediate one $\left(r_{\mathrm{eff}}=\right.$ $2.81 \mathrm{kpc})$ with $n=0.55$, and an outer one $\left(r_{\mathrm{eff}}=4.54 \mathrm{kpc}\right)$ with $n=6.50$.

At the distance of UNAM-KIAS 613, the 3 arcsec fiber from the SDSS spectrum subtends about $1.62 \mathrm{kpc}$. From our spectroscopic analysis, this galaxy initially qualifies as a TO (see Fig. 8) with broad emission lines (see panel d in Fig. A.2). UNAMKIAS 613 is the only galaxy in the sample where a broad component in $\mathrm{H} \alpha$ is clearly detected as can be seen in more detail in Fig. A.3. Also a fainter broad component is detected in $\mathrm{H} \beta$ (see panel d in Fig. A. 2 where the whole spectrum is plotted) and consequently, according to Osterbrock's classification (Osterbrock 1989 ) it corresponds to a type $1.8 \mathrm{AGN}$. The $\mathrm{H} \alpha$ profile shows an extreme, broad double-peaked line. Figure A.3 shows the multicomponent spectral fitting done in the $\mathrm{H} \alpha$ region. In particular, the presence of a broad component in $\mathrm{H} \alpha$ is clear, as well as a double-peaked $\mathrm{H} \alpha$ with two additional broad components, i.e., one component to the blue and the other to the red of the central line. In addition, we have fitted Gaussian functions to the narrow components of $\mathrm{H} \alpha$, [O I]6300,6364, [N II]6548, 6584, and the $[\mathrm{S} \mathrm{II}]$ doublet. This double-peaked profile observed in UNAM-KIAS 613 is similar to that present in a small fraction of AGNs (less than 3\%; Strateva et al. 2003) as is the case in Arp 102B (Popović et al. 2014) and in other double-peaked line emitters detected in the SDSS (Wang et al. 2005). The full width half maximum (FWHM) of the whole broad $\mathrm{H} \alpha$ profile of UNAM-KIAS 613 achieves $\sim 16000 \mathrm{~km} \mathrm{~s}^{-1}$ and the separation between the blue and red broad components is larger than $10000 \mathrm{~km} \mathrm{~s}^{-1}$. Different models have been suggested as possible explanations of the broad double-peaked low ionization emission lines in AGNs, although the preferred interpretation for the measured parameters in the $\mathrm{H} \alpha$ of UNAM-KIAS 613 corresponds to emission from a relativistic accretion disk model (e.g., Eracleous et al. 2009; Gezari et al. 2007). This galaxy is 


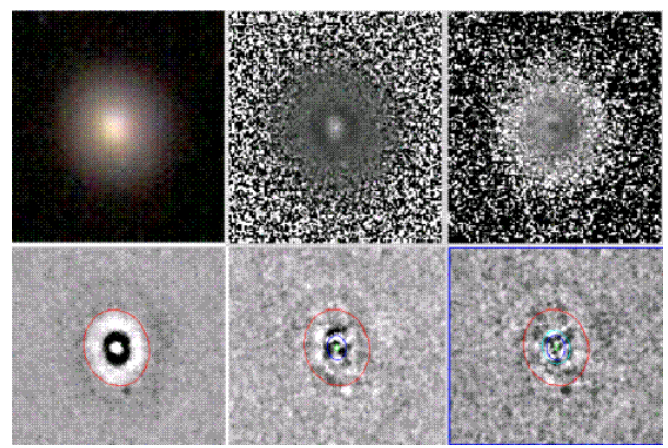

a)

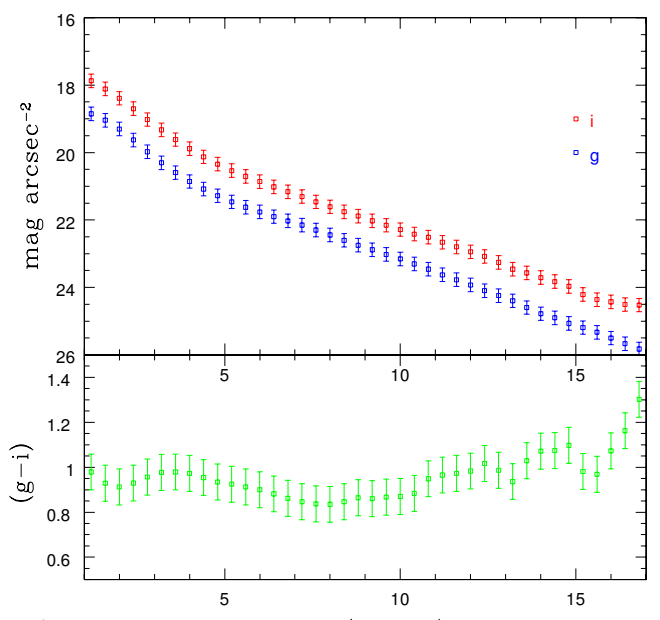

c)

SMA (arcsec)
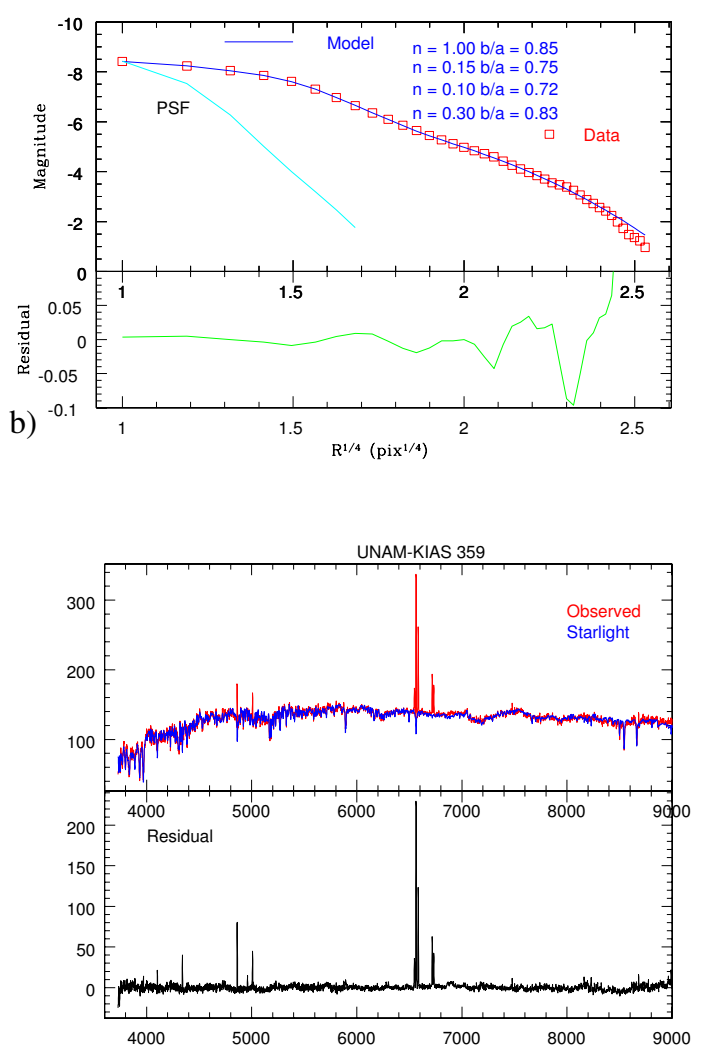

d)

Fig. A.1. Analysis for the blue SF galaxy UNAM-KIAS 359. Top-left panels a): first row shows (from left to right) the SDSS gri-band color image, filter-enhanced $r$-band image, and $g-i$ color map. Second row shows (from left to right) residual $r$-band images from Sérsic1, Sérsic2, and Sérsic3 models. The red ellipse shows the geometric parameters for one Sersic component, whereas additional components are shown in blue, green, and cyan ellipses if they correspond. Top-right panels b): azimuthally averaged surface brightness distribution (red squares) and that derived from the best Sérsic components model (blue line). PSF modeling of field stars is shown as a cyan line. The residual comparison is shown in the lower box (green line). Bottom-left panels c): surface brightness profile in the bands $g$ and $i$ (blue and red squares, respectively). The $g-i$ color gradient is shown in the lower box. Bottom-right panels d): observed SDSS spectrum (red line) along with the corresponding modeled population synthesis spectrum (blue line). The residual spectrum is shown in the lower box (black line).

reported as Seyfert 1.9 in the compilation by Véron-Cetty \& Véron (2006).

\section{A.3. UNAM-KIAS 1197}

No similar-sized neighbor galaxy is found within $692 \mathrm{kpc}$ and $\pm 1000 \mathrm{~km} \mathrm{~s}^{-1}$. Our images in the top-left panel of Fig. A.4 distinguishes a conspicuous bright central region with more uniform blue color along the face of this galaxy and an outer diffuse red region.

The $2 \mathrm{D}$ residual images show some central localized patches, probably associated with dust structures. The sloshing is not significant in the inner kpc. There is evidence of external low surface brightness shell-like structures.

Our analysis from GALFIT (top-right panel of Fig. A.4) shows three components: an inner component $\left(r_{\mathrm{eff}}=0.52 \mathrm{kpc}\right)$ with Sérsic index $n=2.0$, another intermediate component $\left(r_{\mathrm{eff}}=2.05 \mathrm{kpc}\right)$ with $n=0.55$, and an outer component $\left(r_{\mathrm{eff}}=5.50 \mathrm{kpc}\right)$ with $n=0.2$.

At the distance of UNAM-KIAS 1197, a 3 arcsec fiber spectrum subtends $1.92 \mathrm{kpc}$. From our spectroscopic analysis, this galaxy qualifies as a LINER (see Fig. 8). We note the intensity of $\mathrm{N}$ II with respect to $\mathrm{H} \alpha$ in the bottom-right panel of Fig. A.4. This feature is usually associated with Seyfert 2 nuclei. However, the galaxy is reported as a Seyfert 1 with broad
Balmer lines in the AGN compilation by Véron-Cetty \& Véron (2006).

\section{A.4. UNAM-KIAS 1394}

Only two neighbor galaxies fainter than 2.5 mag with respect to UNAM-KIAS 1394 are within $726 \mathrm{kpc}$ and $\pm 1000 \mathrm{~km} \mathrm{~s}^{-1}$. The top-left panel of Fig. A.5 shows a compact nuclear region and a diffuse outer region. The color map shows bluer colors along the face of this galaxy and a clear color gradient in the central region.

Our 2D residual images show a set of localized filaments in the central region, probably associated with dust structures. This galaxy does not show any significant sloshing in the inner kpc. We do not detect any low surface brightness features in this galaxy.

Top-right panel of Fig. A.5 shows two inner components $\left(r_{\text {eff }}=0.60\right.$ and $\left.0.72 \mathrm{kpc}\right)$ with Sérsic indices $n=0.63$ and 1.41 , respectively, and a more extended intermediate component $\left(r_{\text {eff }}=1.71 \mathrm{kpc}\right)$ with $n=1.89$. In this case, a solution with only one inner component in addition to the intermediate component is also reasonable.

At the distance of UNAM-KIAS 1394, the 3 arcsec fiber spectrum subtends $2.1 \mathrm{kpc}$. The spectroscopic analysis shows 
I. Lacerna et al.: Isolated elliptical galaxies in the local Universe

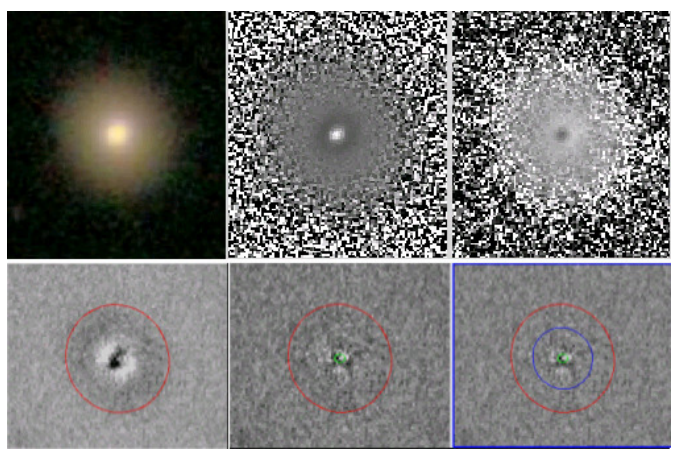

a)
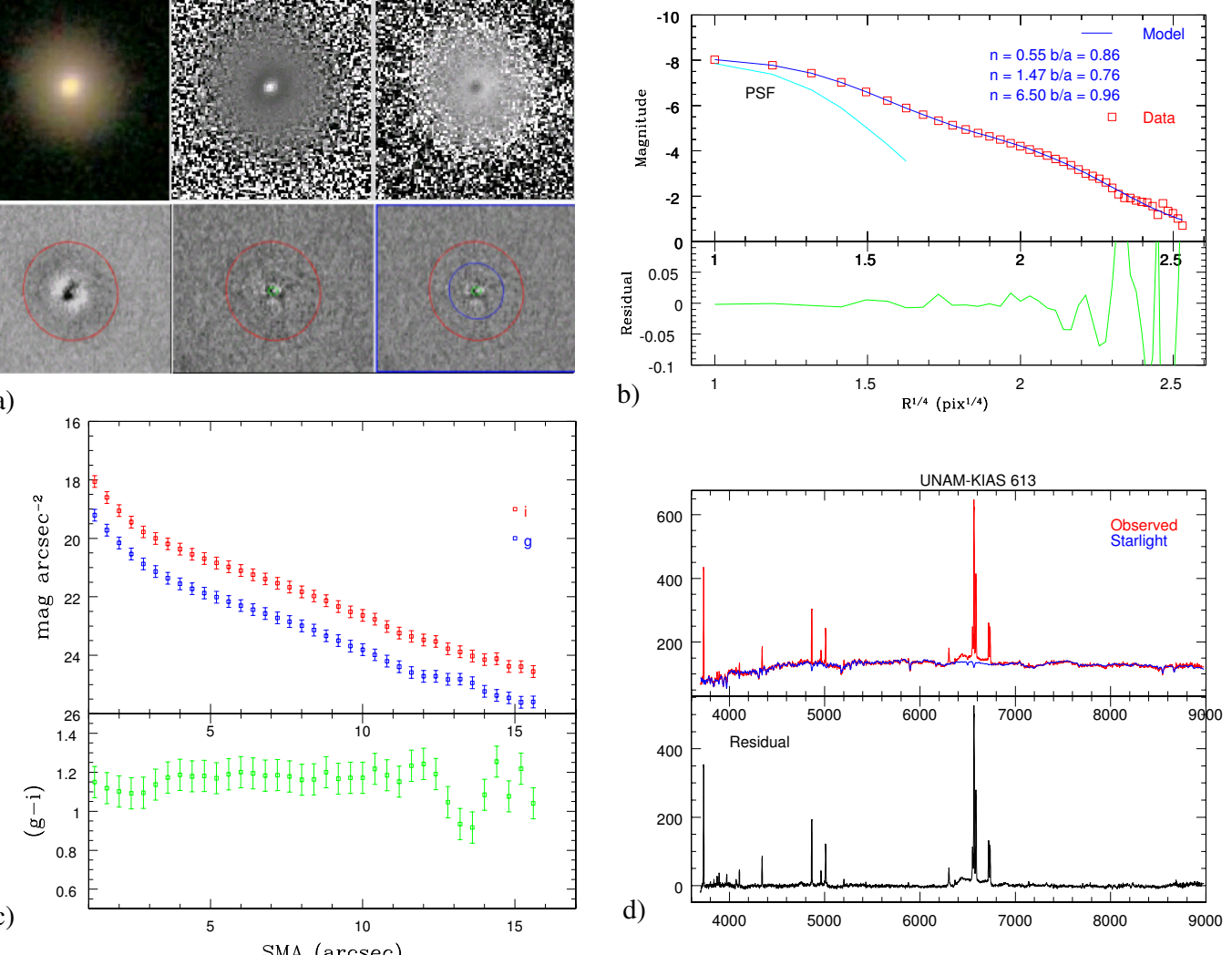

Fig. A.2. Similar to Fig. A.1, but for UNAM-KIAS 613.

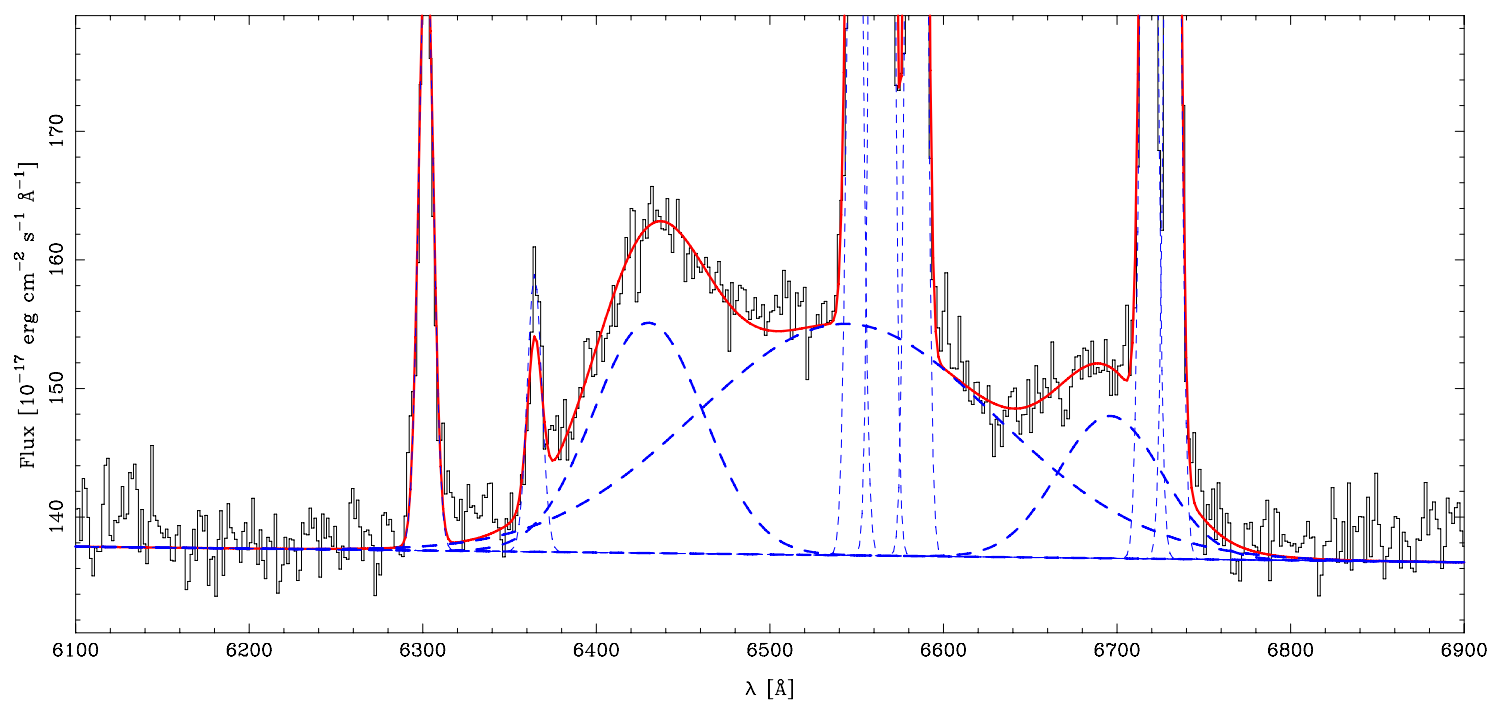

Fig. A.3. Spectrum of the $\mathrm{H} \alpha$ region of UNAM-KIAS 613. Horizontal axis is in the rest-frame wavelength. Black line corresponds to the original host galaxy subtracted spectrum, while the red line shows the model including all the emission-line components. The blue thick dashed lines trace the broad central component and the blue and red broad components of the $\mathrm{H} \alpha$ line, while the blue thin dashed lines show the individual profiles associated with the narrow components (H $\alpha$, [O I]6300,6364, [N II]6548, 6584, [S II]6717, 6731).

definite star formation in the nuclear region (SFN) for this galaxy (see Fig. 8).

\section{Appendix B: Methodology of the photometric analysis}

The homogeneous collection of 0.39 arcsec/pixel optical images available in the SDSS was used to carry out a photometric characterization of our E galaxies, in particular the four blue SF isolated galaxies. Searching for fine structure in the inner/outer regions of elliptical galaxies can be achieved with filter enhancement of the images. This procedure involves taking the original image of the galaxy and filtering it with a Gaussian kernel of two/three times the size of the features to enhance. Filterenhancement was applied to the $r$-band SDSS images. Features in elliptical galaxies can also be revealed by color maps. Since 
A\&A 588, A79 (2016)

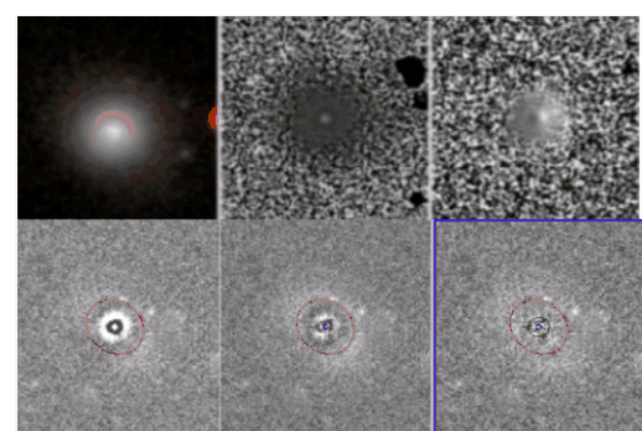

a)

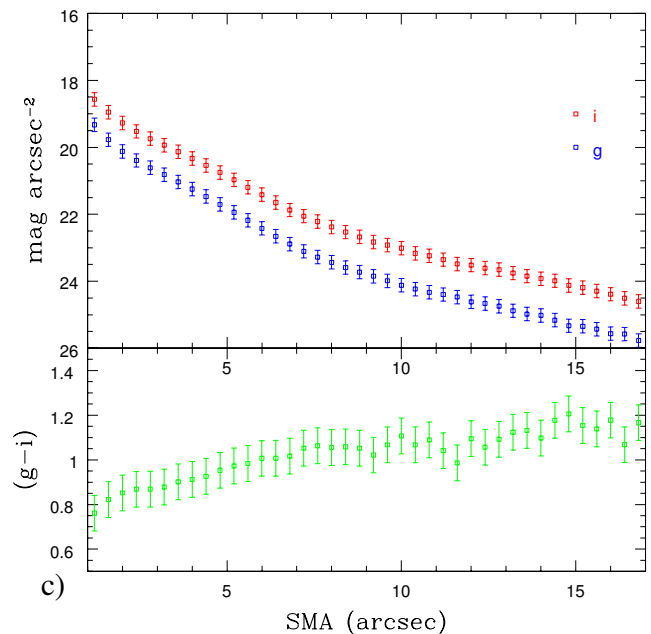

b)

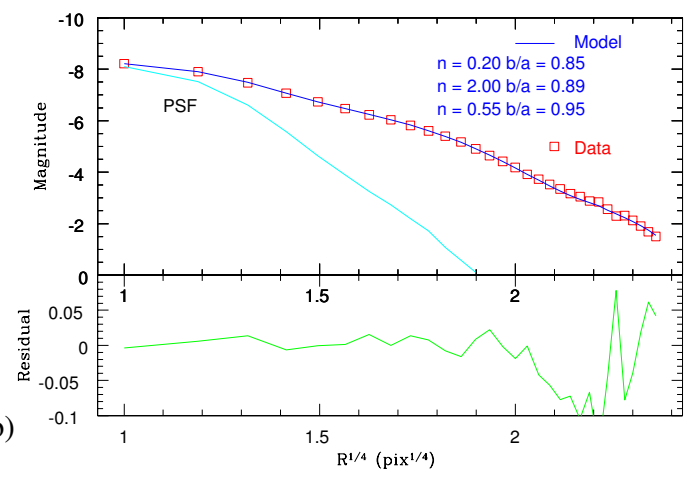

d)

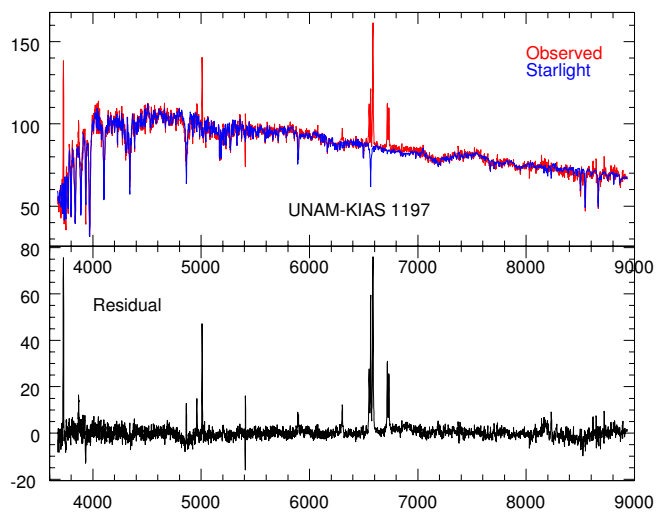

Fig. A.4. Similar to Fig. A.1, but for UNAM-KIAS 1197.
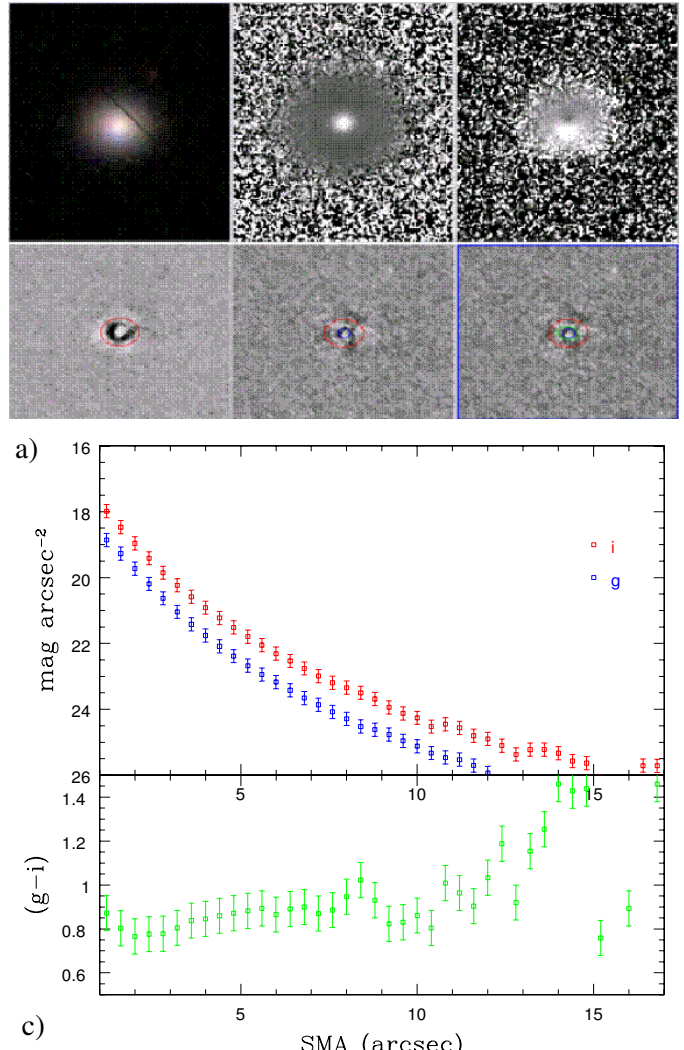

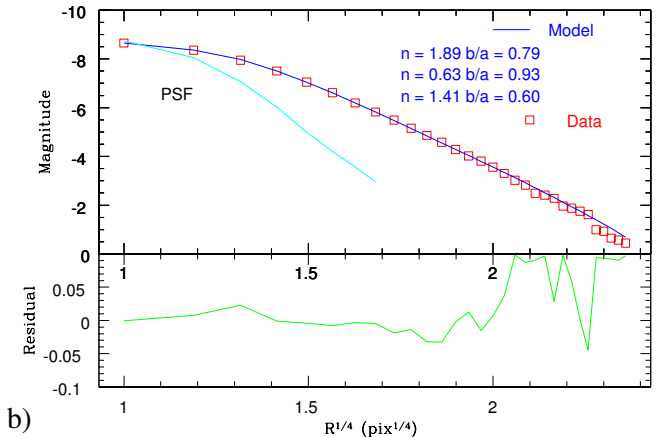

d)

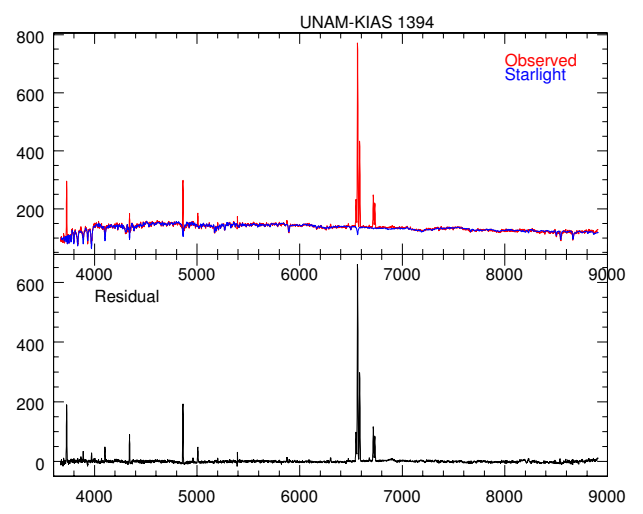

Fig. A.5. Similar to Fig. A.1, but for UNAM-KIAS 1394. 
Table A.1. Photometric properties of the blue SF isolated elliptical galaxies.

\begin{tabular}{lcccc}
\hline \hline Name & Fine structure & Components & $r_{\text {eff }}$ & $n$ \\
\hline UNAM-KIAS 359 & marginal LSB outer shells, & inner & 0.15 & 0.10 \\
& dusty central region & inner & 0.55 & 0.15 \\
& & inner & 0.80 & 1.95 \\
& & intermed & 0.30 & 1.00 \\
UNAM-KIAS 613 & dusty central region & inner & 0.43 & 1.47 \\
& & intermed & 2.81 & 0.55 \\
UNAM-KIAS 1197 & & outer & 4.54 & 6.50 \\
& LSB outer shells, & inner & 0.52 & 2.00 \\
& dusty central region & intermed & 2.05 & 0.55 \\
UNAM-KIAS 1394 & & outer & 5.50 & 0.20 \\
& dusty central region & inner & 0.60 & 0.63 \\
& & inner & 0.72 & 1.41 \\
& & intermed & 1.71 & 1.89 \\
\hline
\end{tabular}

Notes. Columns are: name in the UNAM-KIAS catalog, type of fine structure features found after the 2D image decomposition, components after a $2 \mathrm{D}$ image decomposition along with their corresponding effective radii $(\mathrm{kpc}, h=0.73)$, and Sérsic indices.

Table A.2. Spectroscopic properties of the blue SF isolated elliptical galaxies.

\begin{tabular}{lccccccc}
\hline \hline Name & Fiber scale & Nuclear spectrum & $\log \left(\operatorname{age}_{l w}\right)$ & Error & $\log \left(\operatorname{age}_{m w}\right)$ & Error & $\Delta($ age $)$ \\
\hline UNAM-KIAS 359 & 1.04 & TO & 9.00 & 1.32 & 9.98 & 0.32 & 8.5 \\
UNAM-KIAS 613 & 1.62 & TO(AGN $)$ & 8.95 & 1.63 & 10.03 & 0.31 & 9.8 \\
UNAM-KIAS 1197 & 1.92 & LINER & 8.77 & 1.00 & 9.89 & 0.43 & 7.2 \\
UNAM-KIAS 1394 & 2.10 & SFN & 8.56 & 1.31 & 9.94 & 0.37 & 8.3 \\
\hline
\end{tabular}

Notes. Columns are: name in the UNAM-KIAS catalog, physical scale (kpc, $h=0.73)$ subtended by the 3 arcsec SDSS fiber at the distance of each galaxy, type of spectrum and ionization source associated with the nuclear region from the modeled SDSS spectrum, $\log _{10}$ of the average light-weighted age (in units of yr) and its error, $\log _{10}$ of the average mass-weighted age (in units of yr) and its error, and the star formation timescale (in units of Gyr).

we possess data from the SDDS ugri bands, we generate $g-i$ color maps to look for color features/gradients toward the center.

As the most important procedure in the present section we choose the method of modeling the two-dimensional (2D) light distribution through GALFIT (Peng et al. 2002, 2010a). From the $2 \mathrm{D}$ image decomposition we try to reproduce the observed surface brightness distribution and explore how these local isolated elliptical galaxies may contain photometrically distinct substructures that can shed light on their evolutionary history.

GALFIT can fit an arbitrary number or combination of parametric functions. For the present study, the Sérsic function is adopted because (i) it is appropriate enough to model the main structural components in galaxies and because (ii) it has been widely used in the literature, thus it is useful for further comparisons with published results. Each galaxy is fit with a series of models, each consisting of one to four Sérsic components. These components were ranked by physical size (according to their effective radius, $\left.r_{\text {eff }}\right)$ and were generically designated as inner, intermediate and outer components. As a first order check of our multicomponent fitting, we extracted the azimuthally averaged 1D surface brightness profile from our best 2D models and compared it with the corresponding profile derived from the original data.

PSF images were built by following procedures in the $\mathrm{IRAF}^{7}$ DAOPHOT package. Good estimates of seeing profiles are mandatory since residual errors in the seeing estimate could lead to mismatches between the model and the data. The different components are always fit by sharing the same central position. Since the presence of field stars can introduce potential uncertainty into the GALFIT component models, these stars were

\footnotetext{
7 http://iraf.noao.edu/
}

PSF subtracted or interpolated either close to the galaxy center or in the neighborhood previous to any fitting. At the end of the modeling, we carefully inspect the residual image with the original overlaid.

As a by-product of our image analysis, a measure of the nonaxisymmetry in the surface brightness distribution in the inner $\mathrm{kpc}$ of the UNAM-KIAS isolated elliptical galaxies is presented. The $r$-band images from the SDSS database were analyzed by fitting elliptical isophotes whose centers were first (i) kept fix and then (ii) allowed to vary. A comparison of the centers of the isophotes in (i) and (ii) may reveal a sloshing pattern or spatial variation in the central $\mathrm{kpc}$ that could indicate mass asymmetry and/or a dynamically unrelaxed behavior in the central regions of these galaxies.

Surface brightness profiles in $g$ and $i$ bands were extracted by imposing a fixed center and also fixed ellipticity $\epsilon$ and position angle estimates during our isophotal analysis. This guarantees homogeneous color estimates. The $g-i$ color gradient is corrected for mean galactic extinction.

\section{B.1. Residual features and fine structure}

One of the most direct ways to study the assembly rate of elliptical galaxies is to measure the incidence of fine structure features. In this study, we examined the residual images after a 2D decomposition using additional enhancement procedures. This helped us (i) to identify problems related to poor modeling and also (ii) to identify possible stellar morphology disturbances in various forms. From the latter, we point out shells that have been observed in the stellar component of nearby ellipticals, considered as the result from an accretion of a small companion by 
a massive galaxy (e.g., Dupraz \& Combes 1986; Cooper et al. 2011). These shells vanish after a few Gyr (Schauer et al. 2014), so they are indicators of a recent interaction. Tidal tails are also recognized as linear streams of stellar matter, which is evidence of a dynamically cold component in an accreted companion. Tails that are produced in this manner appear wide and shortlived in comparison to the long and narrow tails produced by interacting spirals. In addition, we note broad fans of stellar light typically of low surface brightness, which are hard to detect in shallow surveys like the SDSS and, finally, highly disturbed galaxies showing signs of an ongoing merger that may be disturbing the stellar component.

Although the more recent multiwavelength surveys suggest that the presence of dust in E/S0 galaxies is the rule rather than the exception, we note here that (i) the distribution of features found in our residual images after a 2D decomposition were presumably associated with dust and that (ii) they are found concentrated on the nucleus and extending out to radii of a few hundred parsecs, rather than spread throughout the galaxy as in other samples (Finkelman et al. 2010).

\section{Appendix C: Methodology of the spectroscopic analysis}

We use the SDSS spectrum of each blue SF isolated elliptical galaxy to infer some stellar population and ionized gas properties. The retrieved SDSS spectra were obtained through an optical fiber of 3 arcsec. Those spectra include not only the nuclear emission but also the absorption bands and continuum produced by the stellar populations of the host galaxy. Thus, it is necessary to subtract the stellar population contribution. This effect is especially important in galaxies of earlier morphological types where the observed spectrum is dominated by the stellar continuum, mainly of G and K-type giant stars, and by middle and old age stellar populations. For this purpose, we have applied STARLIGHT models to the observed SDSS spectra. The models are based on a combination of 45 simple stellar populations (SSPs) from Bruzual \& Charlot (2003) with a Chabrier IMF. The SSP library used here consists of three metallicities Z $(0.004$, 0.02 and 0.05 ) for 15 ages between 0.001 and 13.0 Gyr. The intrinsic extinction was modeled as a uniform dust screen, adopting the extinction law by Cardelli et al. (1989). Spectral fits were carried out in the spectral region between $3000 \AA$ and $9100 \AA$.

After removing the underlying continuum of the galaxy, all resulting spectra were reviewed to identify the presence of emission lines to construct the BPT diagram. In the spectral range

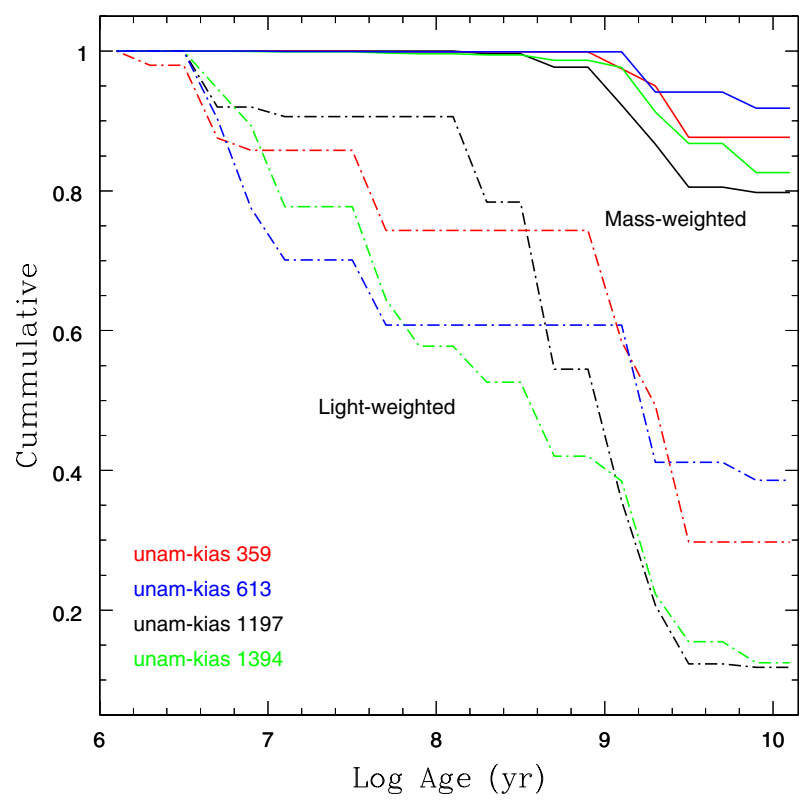

Fig. C.1. Cumulative stellar population synthesis distributions as a function of the lookback time for light-weighted ages (dot-dashed lines) and mass-weighted ages (solid lines) of the blue SF isolated E galaxies (UNAM-KIAS 359 in red, UNAM-KIAS 613 in blue, UNAM-KIAS 1197 in black, and UNAM-KIAS 1394 in green).

covered by the SDSS spectra, the main detected emission lines correspond to $\mathrm{H} \beta$ 4863, [O III]5007, [O I]6300, doublet [N II] 6548, 6584, H $\alpha$ 6583, and sulfur doublet [S II]6717, 6731. The measurement of these lines was carried out interactively by Gaussian fitting with the SPLOT task in IRAF. We also use NGAUSSFIT routine when more than one Gaussian was needed to fit the shape of an emission line, for example, because of the presence of broad line components.

The spectroscopic properties of each galaxy are shown in the bottom-right panels of Figs. A.1, A.2, A.4, and A.5, showing simultaneously (i) the observed spectrum; (ii) the best fit to the observed spectrum from the STARLIGHT model, and, finally; (iii) the residual spectrum after subtracting the observed and best-fit spectrum.

We also applied the STARLIGHT population synthesis models to infer the star formation history (SFH) of these galaxies. The results are presented in Fig. C.1 as the corresponding cumulative distributions of light-weighted and mass-weighted ages of the stellar populations that better reproduce the observed spectra. The average values are reported in Table A.2. 